\begin{tabular}{|c|c|c|}
\hline Beitr. Ent. & Keltern & ISSN 0005-805X \\
\hline $\mathbf{6 1}(2011) 1$ & S. $155-192$ & 16.05 .2011 \\
\hline
\end{tabular}

\title{
Biodiversità delle Aleocharinae della Cina: Athetini, Prima Parte, Generi Lasiosomina, Hydrosmecta, Amischa, Alomaina, Paraloconota, Bellatheta, Nepalota, Pelioptera, Tropimenelytron, Berca e Amphibolusa ${ }^{1}$
}

\section{(Coleoptera, Staphylinidae)}

Con 110 figure

\section{Roberto Pace}

\section{Summary}

Description of 32 new species of the Athetini belonging to the genera Lasiosomina: 1 species (L. weiensis n. sp.), Hydrosmecta: 2 species (H. sichuanensis n. sp., H. sinica n. sp.), Amischa: 1 species (A. sichuanensis n. sp.), Alomaina: 1 species (A. sinica n. sp.), Paraloconota: 6 species (P. shalulimontis n. sp., $P$. daxuemontis n. sp., $P$. erlangensis n. sp., $P$. daxuensis n. sp., $P$. tibetana n. sp., $P$. bilobifera n. sp.) Bellatheta: 4 species (B. tibetana n. sp., B. huamontis n. sp., B. ruficollis n. sp., B. yunnanensis n. sp.), Nepalota: 10 species ( N. erlangensis n. sp., N. yunnanensis n. sp., N. qinlingmontis n. sp., $N$. ruficollis n. sp., N. rougemonti $\mathrm{n}$. sp., $N$. robusta n. sp., $N$. gracilis n. sp., $N$. dabamontis n. sp., $N$. caluoensis n. sp., $N$. daxuensis n. sp.), Pelioptera: 4 species (P. dalicola n. sp., P. dabamontis n. sp., P. shaanxiensis n. sp., P. asymmetrica n. sp.), Tropimenelytron: 1 species (T. qinlingmontis n. sp.), Berca: 1 species (B. sinensis n. sp.), Amphibolusa: 1 species (A. chinensis n. sp.). Every new species is illustrated and compared with similar species. Additional records of 22 species are reported, between them 2 first records from China. The genus Dvorakatheta Maruyama, 2006 is set in synonymy of Paraloconota Cameron, 1939. Three new combinations are given.

Key words

Coleoptera, Staphylinidae, Aleocharinae, taxonomy, China

\section{Zusammenfassung}

Die Arbeit enthält Beschreibungen von 32 neuen Arten der Athetini-Gattungen Lasiosomina: 1 Art (L. weiensis n. sp.), Hydrosmecta: 2 Arten (H. sichuanensis n. sp., H. sinica n. sp.), Amischa: 1 Art (A. sichuanensis n. sp.), Alomaina: 1 Art (Alomaina sinica n. sp.) Paraloconota: 6 Arten (P. shalulimontis n. sp., P. daxuemontis n. sp., P. erlangensis n. sp., P. daxuensis n. sp., P. tibetana n. sp., P. bilobifera n. sp.) Bellatheta: 4 Arten (B. tibetana n. sp., B. huamontis n. sp., B. ruficollis n. sp., B. yunnanensis n. sp.), Nepalota: 10 Arten (N. erlangensis n. sp., $N$. yunnanensis n. sp., $N$. qinlingmontis n. sp., $N$. ruficollis n. sp., $N$. rougemonti $n$. sp., $N$. robusta n. sp., $N$. gracilis n. sp., N. dabamontis n. sp., N. caluoensis n. sp., N. daxuensis n. sp.), Pelioptera: 4 Arten (P. dalicola n. sp., P. dabamontis n. sp., P. shaanxiensis n. sp., P. asymmetrica n. sp.), Tropimenelytron: 1 Art (T. qinlingmontis n. sp.), Berca: 1 Art (B. sinensis n. sp.), Amphibolusa: 1 Art (A. chinensis n. sp.). Jede neue Art wird illustriert und mit ähnlichen Arten vergleichen. Für 22 Arten liegen zusätzlichen Daten vor, u. a. zwei Erstnachweise für China. Die Gattung Dvorakatheta Maruyama, 2006 wird zum Synonym von Paraloconota CAMERon, 1939. Weiterhin werden drei Neukombinationen aufgeführt.

${ }^{1} 246^{\circ}$ Contributo alla conoscenza delle Aleocharinae. 


\section{Riassunto}

Descrizione di 32 nuove specie degli Athetini appartenenti ai generi Lasiosomina: 1 specie (L. weiensis n. sp.), Hydrosmecta: 2 specie (H. sichuanensis n. sp., H. sinica n. sp.), Amischa: 1 specie (A. sichuanensis n. sp.), Alomaina: 1 specie (A. sinica n. sp.) Paraloconota: 6 specie (P. shalulimontis n. sp., P. daxuemontis n. sp., P. erlangensis n. sp., P. daxuensis n. sp., P. tibetana n. sp., P. bilobifera n. sp.), Bellatheta: 4 specie (B. tibetana n. sp., B. huamontis n. sp., B. ruficollis n. sp., B. yunnanensis n. sp.), Nepalota: 10 specie (N. erlangensis n. sp., $N$. yunnanensis n. sp., $N$. qinlingmontis n. sp., $N$. ruficollis n. sp., $N$. rougemonti n. sp., $N$. robusta n. sp., $N$. gracilis n. sp., $N$. dabamontis n. sp., $N$. caluoensis n. sp., $N$. daxuensis n. sp.), Pelioptera: 4 specie (P. dalicola n. sp., P. dabamontis n. sp., $P$. shaanxiensis n. sp., P. asymmetrica n. sp.), Tropimenelytron: 1 specie (T. qinlingmontis n. sp.), Berca: 1 specie (B. sinensis n. sp.), Amphibolusa: 1 specie (A. chinensis n. sp.). Ogni nuova specie è illustrata e comparata con specie simili. Sono riferiti dati aggiuntivi per 22 specie, tra cui 2 primi ritrovamenti per la Cina. Il genere Dvorakatheta Maruyama, 2006 è posto in sinonimia di Paraloconota CAMERoN, 1939. Sono date tre combinazioni nuove.

\section{Introduzione}

In questi ultimi anni le ricerche entomologiche si sono particolarmente intensificate in Cina, con conseguente incremento di pubblicazioni riguardanti lo studio tassonomico di Aleocharinae. In questo ambito si inserisce anche il presente lavoro che è un contributo a una sempre migliore conoscenza sulla tassonomia di questa sottofamiglia degli Staphylinidae della Cina.

\section{Materiale e metodo}

Gli esemplari del presente lavoro sono stati raccolti recentemente $(1997,1999,2001,2003)$ dai colleghi studiosi di Staphylinidae Dr. Aleš Smetana di Ottawa, Guilleaume de Rougemont di London, Michael Schülke di Berlino. Comprende anche le raccolte di A. Pütz a me affidate in studio da Olaf Jäger dello Senckenberg Museum für Tierkunde di Dresda.

Anche lo studio tassonomico delle specie cinesi della sottofamiglia Aleocharinae, come quello d'altre regioni zoogeografiche, presenta seri problemi interpretativi che sono risolti in gran parte grazie all'esame della forma dell'organo copulatore maschile (edeago) e della spermateca. Gli esemplari sono stati dissezionati con lo scopo di includere le strutture genitali in balsamo del Canadà (su piccoli rettangoli trasparenti di materiale di plastica, che accompagnano gli esemplari). Le strutture genitali sono state studiate con l'uso di un microscopio composto fino a 450 ingrandimenti e disegnate per mezzo di oculare a reticolo. Le misurazioni sono state eseguite mediante l'ausilio di un oculare con scala micrometrica. I caratteri dell'habitus sono stati osservati con microscopio stereoscopico fino a 100x. Tutti i disegni delle tavole e le foto sono stati da me eseguiti fino alla fase finale. Le tavole sono state da me composte al computer.

\section{Acronimi}

Gli holotypi e paratypi delle nuove specie sono conservati nei seguenti istituti:

SDEI Senckenberg Deutsches Entomologisches Institut di Müncheberg

SMTD Senckenberg Museum für Tierkunde, Dresden

MNHU Museum für Naturkunde der Humboldt Universität, Berlin

IRSNB Institut royal des Sciences naturelles de Belgique, Bruxelles

Altri paratypi in collezione Smetana. 


\section{Lasiosomina PACE, 1990}

\section{Lasiosomina weiensis n. sp.}

(Figg. 1 e 33-34)

\section{Materiale tipico:}

Holotypus o ${ }^{*}$, China: Shaanxi, Wei He, bank at bridge on autoroute to Xian Airport, $15 \mathrm{~km} \mathrm{~N}$ Xian, 400 m, 34² $4^{\prime} \mathrm{N} 108^{\circ} 55^{\prime} \mathrm{E}, 22$.VIII.1995, leg. A. Pütz (SMTD).

\section{Descrizione:}

Lunghezza 1,6 mm. Avancorpo opaco, addome lucido. Corpo giallo-bruno, capo bruno, margine posteriore delle elitre e i due uroterghi liberi basali gialli, uroterghi liberi terzo, quarto e quinto nero-bruni, antenne giallo-brune con i due antennomeri basali giallo sporco, zampe gialle. Secondo antennomero più corto del primo, terzo più corto del secondo, antennomeri quarto a decimo fortemente trasversi. Occhi lunghi quanto le tempie. Reticolazione di capo e pronoto forte, quella dell'addome assente. Punteggiatura di capo e pronoto indistinta. Granulosità delle elitre finissima e fittissima, quella dell'addome evidente. Un solco trasverso basale profondo sul primo urotergo libero basale, seconda debolmente solcato alla base. Edeago figg. 33-34.

\section{Comparazioni:}

L'habitus della nuova specie è simile a quello di L. lii PACE, 1998 pure della Cina (Hebei Yongnian), specie nota solo sulla + . Se ne distingue per gli occhi lunghi quanto le tempie (occhi più lunghi delle tempie in lii) e per l'addome privo di reticolazione (addome fortemente reticolato in lii).

\section{Etimologia:}

Il nome della nuova specie significa «Della Piccola Baia». Wei in cinese significa piccola baia.

\section{Hydrosmecta Thomson, 1858}

\section{Hydrosmecta perignota PACE, 1998}

Hydrosmecta perignota PACE, 1998: 417

1 đ̋, China: Ying Kou Country, Liaoning Prov., 1987, leg. Li Jing Ke (MNHU).

\section{Distribuzione:}

Specie finora nota solo di Beijing.

\section{Hydrosmecta sichuanensis $\mathrm{n} . \mathrm{sp}$.}

(Figg. 2 e 35-37)

\section{Materiale tipico:}

Holotypus ơ, China, Sichuan, Qingcheng Shan, $65 \mathrm{~km} \mathrm{NW} \mathrm{Chengdu,} \mathrm{ca.} 600 \mathrm{~m}, 10 \mathrm{~km} \mathrm{~W}$ Taiping, 3055'N 10330'E, 4.VI.1997, leg. A. Pütz (SMTD). 
Paratypi: $6 o^{\star} o^{\star}$ e 2 우 옹 stessa provenienza; China: N-Sichuan, Daba Shan, $65 \mathrm{~km}$ N Fengjie, river bank, $1000 \mathrm{~m}, 31^{\circ} 40^{\prime} \mathrm{N} 109^{\circ} 34^{\prime} \mathrm{E}, 10 . \mathrm{VII} .2001$, fine gravel bank (floating treading), leg. M. Schülke (SMTD).

\section{Descrizione:}

Lunghezza 2,27-2,57 mm. Corpo debolmente lucido, appiattito e giallo-bruno, uroterghi liberi dal terzo al quinto bruni, antenne brune con i due antennomeri basali gialli, zampe gialle. Secondo antennomero più corto del primo, terzo lungo quanto il secondo, antennomeri quarto a decimo più lunghi che larghi. Occhi lunghi quanto le tempie. Reticolazione del capo superficiale, quella di pronoto ed elitre forte, quella dell'addome evidente. Punteggiatura di capo, pronoto ed elitre indistinta. Granulosità dell'addome fine e fittissima. Edeago figg. 35-36, spermateca fig. 37.

\section{Comparazioni:}

A motivo della presenza del decimo antennomero più lungo che largo, la nuova specie è simile a $H$. tenuis CAmeron, 1939 dell'India, ma questa specie ha corpo lungo $1,5 \mathrm{~mm}$ e non 2,27-2,57 $\mathrm{mm}$ come nella nuova specie e occhi più corti delle tempie.

\section{Etimologia:}

La nuova specie prende nome dal Sichuan.

\section{Hydrosmecta sinica n. sp.}

(Figg. 3 e 38-39)

\section{Materiale tipico:}

Holotypus ơ, China: Shaanxi, Qin Ling Shan, 3355'N 108 $49^{\circ} \mathrm{E}$, autoroute 50, $40 \mathrm{Km}$ S Xian, riverbank, 1200 m, 31.VIII.1995, leg. A. Pütz (SMTD).

\section{Descrizione:}

Lunghezza 2,18 mm. Corpo lucido e bruno, elitre e uroterghi liberi primo e secondo giallo-bruni, addome bruno, zampe giallo-rossicce. Secondo antennomero più corto del primo, terzo più corto del secondo, antennomeri quarto a decimo più lunghi che larghi. Occhi lunghi quanto le tempie. Reticolazione del capo molto superficiale, quella di pronoto ed elitre evidente, quella dell'addome assente. Punteggiatura del capo finissima, fittissima e superficiale, quella di pronoto ed elitre assente. Granulosità dell'addome fine e fittissima. Un'impressione mediana posteriore trasversa sul pronoto. Primi due uroterghi liberi con solchi basali transversi. Edeago figg. 38-39.

\section{Comparazioni:}

Per la presenza del decimo antennomero più lungo che largo, la nuova specie è simile a $H$. tenuis Cameron, 1939 dell'India. Se ne distingue per la taglia corporea maggiore e il pronoto più lungo che largo (lungo quanto largo in tenuis).

\section{Etimologia:}

Il nome della nuova specie significa «Abitatrice della Cina». 


\section{Leptostiba PaCE, 1985}

\section{Leptostiba alticola (PACE, 2004), comb. n.}

Amischa alticola PACE, 2004: 468

$10^{\star}$, China: Sichuan, Ganzi Prefecture, Daxue Shan, Gongga Shan, Hailougou glacier park, $102^{\circ} 04^{\prime} \mathrm{E} 29^{\circ} 36^{\prime} \mathrm{N}$, river valley, ca. $1 \mathrm{~km}$ above camp I, $2100 \mathrm{~m}, 28-31 . V .1997$, leg. A. Pütz (SMTD).

\section{Distribuzione:}

Specie finora nota solo del Sichuan.

Nota:

Fuorviato dalla forma allargata delle tempie, avevo in precedenza attribuito alticola al genere Amischa, e omessa l'osservazione della forma della ligula. Il ritrovamento del ơ sullo stesso rilievo montuoso, Gongga Shan, e l'osservazione della forma della ligula, portano alla presente nuova attribuzione generica.

\section{Leptostiba miriapex (PACE, 2004), comb. n.}

Atheta (Microdota) miriapex PACE, 2004: 485

1 ơ $^{\star}$, China: W-Sichuan, Yảan Prefecture, Fulin Co., Daxiang Ling, Pass N Hanyuanjie, $51 \mathrm{~km}$ NNE Shimian, smal stream, 2300 m, 10.VII.1999, 29³9'N 102³7'E, leg. A. Pütz (SMTD).

\section{Distribuzione:}

Specie finora nota solo del Sichuan.

Nota:

Fuorviato dalla taglia corporea e dalla forma delle antenne e dell'edeago, avevo in precedenza attribuito alticola al genere Atheta (Microdota), per questo avevo omesso l'osservazione della forma della ligula. Il ritrovamento di nuovo materiale ha permesso la presente attribuzione generica.

\section{Amischa Thomson, 1858}

\section{Amischa sichuanensis n. sp.}

(Figg. 4 e 40)

\section{Materiale tipico:}

Holotypus + , China: W-Sichuan, Yảan Prefecture, Tianquan Co., Jiajin Shan, below Labahe nr. Station, 54 km W Yàan, 1500 m, 3002'90'N 102²6'71"E, 12.VII.1999, leg. A. Pütz (SMTD).

\section{Descrizione:}

Lunghezza 2,12 mm. Corpo lucido e giallo-rossiccio, capo giallo-bruno, antenne brune con i due antennomeri basali e base del terzo gialli, zampe gialle. Secondo antennomero lungo quanto il primo, terzo più corto del secondo, antennomeri quarto a decimo fortemente trasversi. Occhi più corti delle tempie. Reticolazione del corpo assente. Granulosità di capo ed elitre fittissima e superficiale, quella di pronoto e addome assai fitta e saliente. Spermateca fig. 40. 


\section{Comparazioni:}

La nuova specie è simile ad $A$. kashmirica CAmeron, 1939 del Kashmir, di cui ho esaminato la serie tipica di 4 o $^{\star}$ o $^{\text {e }} 2$ 우 우 raccolti a Gulmarg il VI-VII.1931 da Cameron. La spermateca della nuova specie ha forma simile a quella di kashmirica, ma è lunga $0,54 \mathrm{~mm}$, mentre quella di kashmirica è lunga $0,32 \mathrm{~mm}$. Il bulbo distale della spermateca della nuova specie, inoltre, è subsferico, mentre è subcilindrico in kashmirica.

\section{Etimologia:}

La nuova specie prende nome dal Sichuan.

\section{Alomaina PACE, 1991}

\section{Alomaina sinica n. sp.}

(Figg. 5 e 41-43)

\section{Materiale tipico:}

Holotypus 오 China: W-Sichuan, Yảan Prefecture, Tianquan Co., W Erlang Shan Pass, 2700 m, 20.VI.1999, 2951'27"N 102¹5'47'E, leg. A. Pütz (SMTD).

\section{Descrizione:}

Lunghezza 2,87 mm. Corpo lucido e rossiccio, elitre e uroterghi liberi primo e secondo bruno-rossicci, uroterghi liberi terzo, quarto e quinto bruni, antenne brune con i tre antennomeri basali giallo-rossicci, zampe giallo-rossicce. Secondo antennomero più corto del primo, terzo più corto del secondo, antennomeri quarto a decimo trasversi. Occhi lunghi quanto le tempie. Reticolazione del capo superficiale, quella di pronoto ed elitre evidente, quella dell'addome assente. Punteggiatura del capo fitta e superficiale. Granulosità del pronoto fine ed evidente, quella delle elitre saliente e fitta, quella dell'addome molto saliente e fitta. Primi due uroterghi liberi con solchi basali transversi. Spermateca fig. 41.

\section{Comparazioni:}

La nuova specie per la forma della spermateca è simile ad $A$. indica PACE, 1991. Se ne distingue per il bulbo distale della spermateca meno dilatato di quello di indica, per la parte intermedia della stessa spermateca lunga 0,22 mm e non 0,12 come in indica. L'addome della nuova specie, inoltre, è privo di reticolazione, mentre quello di indica è coperto di forte reticolazione.

\section{Etimologia:}

Il nome della nuova specie significa «Abitatrice della Cina».

\section{Ischnopoda STEPHens, 1835}

\section{Ischnopoda wei (PACE, 1993)}

Tachyusa (Caliusa) wei PACE, 1993: 88

Ischnopoda wei: SMETANA, 2004: 490

1 ㅇ , China: Sichuan, Leshan, X.1985, leg. G. de Rougemont (IRSNB). 


\section{Distribuzione:}

Specie finora nota solo dell'Emei Shan.

\section{Ischnopoda hebeiensis PACE, 1998}

Ischnopoda (Caliusa) hebeiensis PACE, 1998: 404

Ischnopoda hebeiensis: SMETANA, 2004: 490

4 es., China: Shaanxi, Wei He, bank at bridge on autoroute to Xian Airport, $15 \mathrm{~km} \mathrm{~N}$ Xian, $400 \mathrm{~m}, 34^{\circ} 24^{\prime} \mathrm{N} 108^{\circ} 55^{\prime} \mathrm{E}, 22$.VIII.1995, leg. A. Pütz (MNHU).

\section{Distribuzione:}

Specie finora nota solo di Hebei Beidaihe, Cina.

\section{Paraloconota CAMERon, 1939}

Paraloconota CAMERon, 1939: 293

Dvorakatheta MaruYama, 2006: 191, n. syn.

\section{Nota sinonimica:}

Ho esaminato sulla serie tipica la specie tipo del genere Paraloconota CAMEron, 1939, P. muscicola Cameron, 1939 (Museo di Londra). Il nuovo genere Dvorakatheta Maruyama, 2006 è stato decritto e illustrato accuratamente. In base alla descrizione e alla parte illustrativa date da Maruyama mi è stato facile e immediato riconoscere che Dvorakatheta Maruyama è sinonimo di Paraloconota Cameron. L'edeago sia in Paraloconota che in Dvorakatheta è diviso in due lobi apicali, la ligula ha forma identica, e la forma allungata delle zampe e delle antenne si ritrova nei due generi. Maruyama non cita Paraloconota come genere da comparare.

\section{Nuova combinazione:}

La nuova combinazione per la specie wrasei Dvořák, 1988 è la seguente: Paraloconota wrasei (DVOŘÁK, 1988), comb. n.

\section{Paraloconota gansuensis PACE, 1998}

Paraloconota gansuensis PACE, 1998: 436

7 es., China: Sichuan, Ganzi Prefecture, Daxue Shan, Gongga Shan, Hailougou glacier park, $102^{\circ} 04^{\prime} \mathrm{E} 29^{\circ} 36^{\prime} \mathrm{N}$, river valley, ca. $1 \mathrm{~km}$ above camp I, $2100 \mathrm{~m}, 28-31 . V .1997$, leg. A. Pütz (SMTD); 5 es., China: Sichuan, Gongga Shan, Hailougou glacier park, env. camp II, 2650 m, 30.V.1997, sifted, leg. A. Pütz (SMTD); 1 \& , China: Sichuan, Daxue Shan, Gongga Shan, Hailougou glacier park, env. glacier tongue, ca. $3,5 \mathrm{~km}$ above camp III, ca. $3200 \mathrm{~m}$, 29.V.1997, leg. A. Pütz (SMTD); 1 + , China: Sichuan, Ganzi Tibetian Auton. Prefecture, Daxue Shan, $10 \mathrm{~km}$ $S$ Kanding, 29 $59^{\prime} \mathrm{N}, 101^{\circ} 55^{\prime} \mathrm{E}, 3150$ m, 26.V.1999, leg. A. Pütz (SMTD); 1 o $^{\mathrm{t}}$ e 1 \% , China: W-Sichuan, Ganzi Tibetian Auton. Pref., Daxue Shan, Mugecuo, 3700 m, 15 km W Kangding,

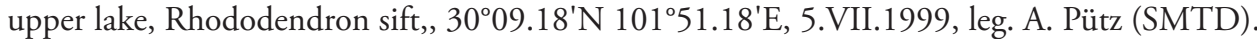

\section{Distribuzione:}

Specie finora nota solo del Gansu. 


\section{Paraloconota shalulimontis n. sp.}

(Figg. 6 e 44-46)

\section{Materiale tipico:}

Holotypus ơ , China: Sichuan, Ganzi Tibet. Aut. Pref. Litang Co., Shaluli Shan, Abies-ForestRest, 25 km NN Litang, 4300 m, 30¹7.23'N 90³0.97'E, 1.VII.1999, leg. A. Pütz (SMTD).

Paratypi: 32 es., stessa provenienza; 16 es., China: Prov. Sichuan, Ganzi Tibet. Aut. Pref. Yajiang Co., Shaluli Shan, E Pass, 15 km W Yajiang, 4300 m, 3000.24'N 10051.63'E, 2.VII.1999, leg. A. Pütz (SMTD).

\section{Descrizione:}

Lunghezza 4,39 mm. Corpo lucido e nero, antenne nere con i quattro antennomeri basali brunorossicci, zampe rossicce con femori bruno-rossicci. Secondo antennomero più corto del primo, terzo più lungo del secondo, antennomeri quarto e quinto più lunghi che larghi, sesto e settimo lunghi quanto larghi, ottavo a decimo trasversi. Occhi più corti delle tempie. Reticolazione dell'avancorpo forte, quella dell'addome molto trasversa e netta. Punteggiatura del capo fine e superficiale, assente sulla fascia longitudinale mediana. Granulosità del pronoto fitta ed evidente, quella delle elitre molto superficiale. Punteggiatura dell'addome fine e rada. Debole solco longitudinale mediano del capo. Edeago figg. 44-45, spermateca fig. 46.

\section{Comparazioni:}

La nuova specie è simile a $P$. fengicola Pace, 1998 del Gansu. Se ne distingue per l'edeago ampiamente arcuato al lato ventrale e per gli antennomeri ottavo a decimo trasversi (più lunghi che larghi in fengicola).

\section{Etimologia:}

La nuova specie prende nome dal Monte Shaluli.

\section{Paraloconota daxuemontis n. sp.}

(Figg. 7 e 47-48)

\section{Materiale tipico:}

Holotypus ơ, China: W-Sichuan, Ganzi Tibetian Auton. Pref., Daxue Shan, Mugecuo, 3700 m, 15 km W Kangding, upper lake, Rhododendron sift,, 3009.18'N 10151.18'E, 5.VII.1999, leg. A. Pütz (SMTD).

\section{Descrizione:}

Lunghezza 4,54 mm. Corpo lucido e nero, antenne nere con i due antennomeri basali nerobruni, zampe rossicce. Secondo antennomero più corto del primo, terzo più corto del secondo, antennomeri quarto a sesto lunghi quanto larghi, settimo a decimo trasversi. Occhi lunghi quanto le tempie. Reticolazione dell'avancorpo molto superficiale, quella dell'addome assente, tranne che sul quinto urotergo libero molto trasversa e assai superficiale. Punteggiatura di capo e pronoto fitta e superficiale. Granulosità delle elitre molto evanescente. Edeago figg. 47-48.

\section{Comparazioni:}

Per la forma dell'edeago e della notevole lunghezza dell'undicesimo antennomero, la nuova specie è simile a P. fengicola PACE, 1998 del Gansu. Se ne distingue per l'edeago angoloso al 
lato ventrale (senza angolo in fengicola) per la "crista proximalis» corta (molto sviluppata in fengicola) e per gli antennomeri quarto a sesto lunghi quanto larghi e non molto più lunghi che larghi come in fengicola.

\section{Etimologia:}

La nuova specie prende nome dal Monte Daxue.

\section{Paraloconota erlangensis n. sp.}

(Figg. 8 e 49-52)

\section{Materiale tipico:}

Holotypus ơ , China: W-Sichuan, Ya’an Prefecture, Tianquan Co., E Erlang Shan Pass, 2900 m, 20.VI.1999, 2952'36"N 102¹7'82"E, leg. A. Pütz (SMTD).

Paratypi: $1 \sigma^{\text {t }}$ e 2 우 우, stessa provenienza.

\section{Descrizione:}

Lunghezza 4,24 mm. Corpo lucido e nero, antenne e zampe rossicce. Secondo antennomero più corto del primo, terzo più lungo del secondo, antennomeri quarto a nono molto più lunghi che larghi, decimo poco più lungo che largo. Occhi più corti delle tempie. Reticolazione di capo e pronoto forte, quella delle elitre distinta, quella dell'addome molto trasversa e ben visibile. Punteggiatura di capo, pronoto ed elitre indistinta. Granulosità dell'addome fine. Edeago figg. 50-51, spermateca fig. 52 .

\section{Comparazioni:}

L'edeago della nuova specie è simile a quello di P. gansuensis PACE, 1998 pure del Sichuan. Se ne distingue per il profilo ventrale dell'edeago ampiamente sinuoso (con profilo ventrale strettamente arcuato e verso l'apice quasi rettilineo in gansuensis).

\section{Etimologia:}

La nuova specie prende nome dal Monte Erlang.

\section{Paraloconota daxuensis n. sp.}

(Figg. 9 e 53-54)

\section{Materiale tipico:}

Holotypus ơ , China: Sichuan, Ganzi Pref., Daxue Shan, $30^{\circ} 03^{\prime} \mathrm{N} 102^{\circ} 00^{\prime} \mathrm{E}$, River Valley, $5 \mathrm{~km}$ E Kangding, 3000 m, 20/23.V.1997, leg. A. Pütz (SMTD).

\section{Descrizione:}

Lunghezza 3,93 mm. Corpo lucido e nero-bruno, antenne bruno-rossicce con antennomeri ottavo a undicesimo bruni, zampe rossicce. Secondo antennomero più corto del primo, terzo più lungo del secondo, antennomeri quarto a decimo molto più lunghi che larghi. Occhi più corti delle tempie. Reticolazione di capo e pronoto forte, quella delle elitre evidente e quella dell'addome molto trasversa ben visibile. Punteggiatura di capo e pronoto assente, quella dell'addome fine. Granulosità delle elitre superficiale. Capo con impressione longitudinale discale. Pronoto con appiattimento mediano. Edeago figg. 53-54. 


\section{Comparazioni:}

L'edeago della nuova specie è profondamente arcuato al lato ventrale e ha un'armatura genitale interna plurilobata. Questi caratteri non sono presenti in P. yonghaiensis PACE, 1998 del Gansu, sua specie simile. Il pronoto di yonghaiensis mostra due profonde fossette, assenti sul pronoto della nuova specie.

\section{Etimologia:}

La nuova specie prende nome dal Monte Daxue.

\section{Paraloconota tibetana n. sp.}

(Figg. 10 e 55-57)

\section{Materiale tipico:}

Holotypus + , China: W-Sichuan, Aba Tibetian Aut. Pref. Weizhou Co., Qionglai Shan, Wolong Valley, 89 km WSW Guanxian, 3900 m, 3053.57'N 10254.67'E, 15.VII.1999, leg. A. Pütz (SMTD).

Paratypi: 10 es., stessa provenienza (SMTD).

\section{Descrizione:}

Lunghezza 5,7 mm. Corpo lucido e nero, antenne brune con i due antennomeri basali brunorossicci, zampe bruno-rossicce con femori bruni. Secondo antennomero più corto del primo, terzo più lungo del secondo, antennomeri quarto a decimo molto più lunghi che larghi. Occhi più corti delle tempie. Reticolazione di capo e pronoto forte, quella delle elitre evidente e quella dell'addome molto trasversa e distinta. Punteggiatura di capo e pronoto indistinta, quella dell'addome fine e rada. Granulosità delle elitre fitta e assai superficiale. Disco del capo impresso. Edeago figg. 55-56, spermateca fig. 57.

\section{Comparazioni:}

L'edeago della nuova specie presenta apice distale, in visione laterale, assai dilatato e appuntito. Questo carattere non si trova nell'edeago delle specie sue simili della Cina e dell'Himalaya Anche la spermateca, robusta e con bulbo distale di forma inconsueta, mostra caratteri unici nell'ambito del genere.

\section{Etimologia:}

La nuova specie prende nome dal Tibet.

\section{Paraloconota bilobifera n. sp.}

(Figg. 11 e 58-60)

\section{Materiale tipico:}

Holotypus ơ , China: Sichuan, Ganzi Pref., Daxue Shan, $30^{\circ} 03^{\prime} \mathrm{N} 102^{\circ} 00^{\prime} \mathrm{E}$, River Valley, $5 \mathrm{~km}$ E Kangding, river valley, ca. 3000 m, 20/23.V.1997, leg. A. Pütz (SMTD).

\section{Descrizione:}

Lunghezza 3,93 mm. Corpo lucido e nero, antenne nero-brune, zampe bruno-rossicce con femori bruni. Secondo antennomero più corto del primo, terzo più lungo del secondo, antennomeri 
quarto a decimo molto più lunghi che larghi. Occhi più corti delle tempie. Reticolazione del capo con aspetto rugoso ed evidente, quella del pronoto forte, quella delle elitre evidente e quella dell'addome molto trasversa. Punteggiatura di capo e pronoto indistinta. Granulosità delle elitre fitta e superficiale. Debole solco mediano discale del pronoto. Edeago figg. 58-59, spermateca fig. 60.

\section{Comparazioni:}

L'apice dell'edeago diviso in due corti e larghi lobi, in visione ventrale, non si è finora mai osservato simile in altre speccie.

\section{Etimologia:}

Il nome della nuova specie significa «Portatrice di due lobi», quelli apicali dell'edeago.

\section{Bellatheta Roubal, 1928}

Nota:

Il genere Emmelostiba PACE, 1982 é stato recentemente posto in sinonimia di Bellatheta RoubaL (Vogel, 2006).

\section{Bellatheta tibetana n. sp.}

(Figg. 12 e 61-63)

\section{Materiale tipico:}

Holotypus ơ, China: W-Sichuan, Ganzi Tibetian Auton. Prefecture, Daxue Shan, river valley, 5 km E Kanding, 3003'N, 10100'E, 2500-2600 m, 24.VI.1999, leg. A. Pütz (SMTD).

Paratypi: 6 es., stessa provenienza; 1 을 China: W-Sichuan, Ganzi Tibetian Auton. Prefecture, Daxue Shan, Mugecuo, $15 \mathrm{~km} \mathrm{NW}$ Kanding, below lowest lake, 3009.18'N, $101^{\circ} 51.16^{\prime} \mathrm{E}$, 3300 m, 27.VI.1997, leg. A. Pütz (SMTD).

\section{Descrizione:}

Lunghezza 2,33 mm. Corpo lucido e bruno, addome nero, antenne brune con i due antennomeri basali bruno-rossicci, zampe giallo-brune con femori bruni. Secondo antennomero più corto del primo, terzo più corto del secondo, quarto più lungo che largo, antennomeri quinto a settimo lunghi quanto larghi, ottavo a decimo trasversi. Occhi molto più corti delle tempie. Reticolazione di capo e pronoto irregolare ed evidente, quella delle elitre ben visibile, quella dell'addome debolmente trasversa e superficiale. Punteggiatura del capo fitta e assai superficiale, quella delle elitre fittissima ed evidente, quella dell'addome superficiale. Granulosità del pronoto fitta e assai superficiale. Pronoto con largo solco mediano. Edeago figg. 61-62, spermateca fig. 63, composta di chitina nero-bruna.

\section{Comparazioni:}

Per la forma dell'edeago e dell'habitus, la nuova specie è affine a $B$. brachycephala (CAMERon, 1939) dell'India, di cui ho esaminato l'holotypus ơ raccolto a Gahan il IX.1921 da Cameron (Museo di Londra). La nuova specie se ne distingue per l'edeago ampiamente arcuato al lato ventrale e senza lobo ventrale (non arcuato in brachycephala e con un lobo ventrale).

\section{Etimologia:}

La nuova specie prende nome dal Tibet. 


\section{Bellatheta huamontis n. sp.}

(Figg. 13 e 64-65)

\section{Materiale tipico:}

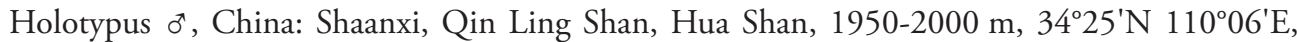
19.VIII.1995, sifted, leg. A. Pütz (SMTD).

\section{Descrizione:}

Lunghezza 2,57 mm. Corpo nero, antenne comprese, zampe brune con tarsi giallo-bruni. Secondo antennomero più corto del primo, terzo più corto del secondo, antennomeri quarto a decimo trasversi. Occhi più corti delle tempie. Reticolazione del disco del capo evidente, quella sul resto della sua superficie assai superficiale. Reticolazione del pronoto evidente, quella delle elitre forte, quella dei tre uroterghi liberi basali molto evanescente, quella degli uroterghi liberi quarto e quinto poco trasversa e superficiale. Punteggiatura dell'avancorpo molto superficiale, sul capo assente sulla fascia longitudinale mediana, quella dell'addome fine ed evidente. Edeago figg. 64-65.

\section{Comparazioni:}

Per la forma dell'edeago la nuova specie è simile a B. franzi (PACE, 1982) del Nepal. Ma l'apice dell'edeago è privo di dentino ventrale presente in franzi e l'armatura genitale interna dell'edeago è corta e larga, mentre è stretta e lunghissima in franzi. Le elitre in franzi, inoltre, sono più corte del pronoto e non più lunghe del pronoto come nella nuova specie.

\section{Etimologia:}

Il nome della nuova specie significa «Del Monte Hua».

\section{Bellatheta ruficollis $\mathrm{n} . \mathrm{sp}$.}

(Figg. 14 e 66-67)

\section{Materiale tipico:}

Holotypus $0^{\star}$, China: N-Yunnan, Dali Bai Nat. Aut. Pref., Diancang Shan, $3 \mathrm{~km}$ W Dali, $25^{\circ} 41.1^{\prime} \mathrm{N} 100^{\circ} 06.8 \mathrm{E}, 29$.VIII.2003, leg. A. Smetana (SDEI).

\section{Descrizione:}

Lunghezza 2,6 $\mathrm{mm}$. Corpo lucido, capo nero, pronoto, primo urotergo libero basale, margine posteriore del secondo e pigidio giallo-rossicci, elitre giallo-brune, antenne brune con i due antennomeri basali e l'undicesimo giallo-rossicci, zampe gialle. Secondo antennomero più corto del primo, terzo più corto del secondo, antennomeri quarto a settimo lunghi quanto larghi, ottavo a decimo trasversi. Occhi lunghi quanto le tempie. Reticolazione dell'avancorpo molto superficiale, quella dell'addome assente. Punteggiatura del capo fortissima e assente nella depressione mediana posteriore, quella di pronoto ed elitre superficiale. Edeago figg. 66-67.

\section{Comparazioni:}

Per il colore giallo-rossiccio del pronoto e per i rami apicali ventrali dell'edeago molto divergenti e stretti la nuova specie è notevolmente differente da tutte le specie note. La presente è la prima specie che presenta pronoto giallo-rossiccio.

\section{Etimologia:}

Il nome della nuova specie significa «Pronoto rossiccio». 


\section{Bellatheta yunnanensis n. sp.}

(Figg. 15 e 68)

\section{Materiale tipico:}

Holotypus + , China: N-Yunnan, Zhongdian Co., $48 \mathrm{~km} \mathrm{~N}$ Zhongdian, $28^{\circ} 16.6^{\prime} \mathrm{N} 99^{\circ} 45.7 \mathrm{E}$, 3220 m, 21.VIII.2003, leg. A. Smetana (SDEI).

\section{Descrizione:}

Lunghezza $4 \mathrm{~mm}$. Corpo lucido e nero, elitre brune, antenne nere con i due antennomeri basali bruni, zampe giallo-rossicce. Secondo antennomero più corto del primo, terzo più lungo del secondo, quarto molto più lungo che largo, quinto poco più lungo che largo, sesto lungo quanto largo, antennomeri settimo a decimo trasversi. Occhi più corti delle tempie. Reticolazione di capo ed elitre evidente, quella del pronoto forte, quella dell'addome assente. Punteggiatura del capo quasi indistinta. Granulosità del pronoto evidente, quella delle elitre superficiale. Spermateca fig. 68.

\section{Comparazioni:}

La spermateca della nuova specie ha forma simile a quella della spermateca di $B$. pahangensis (CAmeron, 1939) della Malaysia, di cui ho esaminato la serie tipica di $10^{\star}$ e 1 ㅇ (Mus. Londra). La nuova specie si distingue per la parte prossimale della spermateca molto più lunga di quella di pahangensis e per la profonda introflessione apicale del bulbo distale. La reticolazione del pronoto della nuova specie è forte, quella del pronoto di pahangensis è assente.

\section{Etimologia:}

La nuova specie prende nome dallo Yunnan.

\section{Nepalota PACE, 1987}

\section{Nepalota smetanai PACE, 1998}

(Figg. 23 e 88-90)

Nepalota smetanai PACE, 1998: 495

15 es., China: Shaanxi, Qin Ling Shan, Hua Shan Mt. N Valley, 1200-1400 m, 118 Km E Xian, $34^{\circ} 27^{\prime} \mathrm{N} 110^{\circ} 06^{\prime} \mathrm{E} 18-20$.VIII.1995, sifted, leg. A. Pütz; 8 es., China: Shaanxi, Qin Ling Shan, $33^{\circ} 45^{\prime} \mathrm{N} 107^{\circ} 56^{\prime} \mathrm{E}$, autoroute Km 93 S of Zhouzhi, $108 \mathrm{Km}$ SW Xian, mountain forest, sifted, 1650 m, 1-2.IX.1995, leg. A. Pütz.; 7 es., China: Shaanxi, Qin Ling Shan, Hua Shan, 1950$2000 \mathrm{~m}, 34^{\circ} 25^{\prime} \mathrm{N} 110^{\circ} 06^{\prime} \mathrm{E}, 19 . \mathrm{VIII} .1995$, sifted, leg. A. Pütz; $30^{\star} 0^{\star}$, China: S-Shaanxi, Qin Ling Shan, mountain range W pass on rd. Xi'an, Shagoujie, $45 \mathrm{~km} \mathrm{SSW} \mathrm{Xi'an,} 2675 \mathrm{~m}, 33^{\circ} 52^{\prime} \mathrm{N}$ 108 46'E, 26.VII.2001, leg. A. Smetana; $10^{\star}$, China: N-Yunnan, Dali Bai Nat. Aut. Pref., Diancang Shan, 3 km W Dali, 2541.1'N 10006.6'E, 2750 m, 1.IX.2003, leg. A. Smetana; 7 es., China: N-Yunnan, Dali Bai Nat. Aut. Pref., Diancang Shan, $3 \mathrm{~km} \mathrm{~W}$ Dali, $25^{\circ} 41.1^{\prime} \mathrm{N} 100^{\circ} 06.6^{\prime} \mathrm{E}$, 2600-2650 m, 30.VIII.2003, leg. A. Smetana; 15 es., China: N-Yunnan, Dali Bai Nat. Aut. Pref., Diancang Shan, 4 km W Dali, 2540.4'N 10006.7'E, 2900-3000 m, 31.VIII.2003, leg. A. Smetana; 13 es., China: Shaanxi, Daba Shan, mtn. range N pass $22 \mathrm{~km} \mathrm{NW} \mathrm{Zhenping,} \mathrm{N}$ slope, $2685 \mathrm{~m}, 32^{\circ} 01^{\prime} \mathrm{N} 109^{\circ} 21^{\prime} \mathrm{E}, 13 . \mathrm{VII} .2001$, leg. A. Smetana; $10^{*}$, China: Shaanxi, Daba Shan, mtn. range $\mathrm{N}$ pass $22 \mathrm{~km} \mathrm{NW}$ Zhenping, $2685 \mathrm{~m}, 32^{\circ} 01^{\prime} \mathrm{N} 109^{\circ} 21^{\prime} \mathrm{E}, 14 . \mathrm{VII} .2001$, leg. 
A. Smetana; $10^{\star}$ e 4 우 을 China: W-Hubei, Daba Shan pass E of Mt. Da Shennongjia, $12 \mathrm{~km}$ NW Muyuping, 19.VII.2001, 1950 m, leg. A. Smetana; 1 ơ $^{\star}$ China: N-Yunnan, Dali Bai Nat. Aut. Pref., Diancang Shan, $3 \mathrm{~km} \mathrm{~W}$ Dali, $25^{\circ} 41.1^{\prime} \mathrm{N} 100^{\circ} 07.8 \mathrm{E}, 29 . V I I I .2003$, leg. A. Smetana (SMTD) (MNHU) (IRSNB).

\section{Distribuzione:}

Specie già nota del Sichuan e Gansu.

\section{Nepalota gansuensis PACE, 1998}

Nepalota gansuensis PACE, 1998: 493

$10^{\star}$, China: N-Yunnan, Zhongdian Co., 33 km ESE Zhongdian, 2741.5'N 100 00.7'E, $3200 \mathrm{~m}$, 24.VIII.2003, leg. A. Smetana; 1 ㅇ China: W-Hubei, Daba Shan pass E of Mt. Da Shennongjia, 12 km NW Muyuping, 19.VII.2001, 1950 m, leg. A. Smetana; $10^{\star}$, China: N.Yunnan, Lijiang, Naxi Aut. Co., E Yulongxue Shan, $30 \mathrm{~km} \mathrm{~N} \mathrm{Lijiang,} 27^{\circ} 09.0^{\prime} \mathrm{N} 100^{\circ} 14.9^{\prime} \mathrm{E}, 2800-2900 \mathrm{~m}$, 13.VIII.2003, leg. A. Smetana; 1 , China: N-Yunnan, Zhongdian Co., 36 km ESE Zhongdian, $27^{\circ} 40.9^{\prime} \mathrm{N} 100^{\circ} 01.5 \mathrm{E}, 3500 \mathrm{~m}, 23 . \mathrm{VIII} .2003$, leg. A. Smetana (MNHU) (IRSNB).

\section{Distribuzione:}

Specie finora nota solo del Gansu.

\section{Nepalota globifera PACE, 1998}

Nepalota globifera PACE, 1998: 945

1 ․ China: N-Yunnan, Dali Bai Nat. Aut. Pref., Diancang Shan, 3 km W Dali, $25^{\circ} 41.1$ 'N $100^{\circ} 07.8$ E, 29.VIII.2003, leg. A. Smetana (MNHU).

\section{Distribuzione:}

Specie già nota dello Yunnan.

\section{Nepalota chinensis PACE, 1998}

Nepalota chinensis PACE, 1998: 945

$10^{\star}$, China: W-Hubei, Daba Shan creek valley, $11 \mathrm{~km} \mathrm{NW}$ Muyuping, $31^{\circ} 30^{\prime} \mathrm{N} 110^{\circ} 22^{\prime} \mathrm{E}$, 1960 m, 18.VII.2001, leg. A. Smetana; 1 ㅇ, China: N-Yunnan, Zhongdian Co., 36 km ESE Zhongdian, $27^{\circ} 40.9^{\prime} \mathrm{N} 100^{\circ} 01.5 \mathrm{E}, 3500 \mathrm{~m}$, 23.VIII.2003, leg. A. Smetana (MNHU) (IRSNB).

\section{Distribuzione:}

Specie finora nota solo dello Yunnan.

\section{Nepalota erlangensis n. sp.}

(Figg. 69-70)

\section{Materiale tipico:}

Holotypus ơ, China: W-Sichuan, Ya'an Prefecture, Tianquan Co., W Erlang Shan, Pass 2900 m, 21.VI.1999, 2951'13"'N 102¹7'28"'E, sifted, leg. A. Pütz (SMTD). 


\section{Descrizione:}

Lunghezza 4,54 mm. Avancorpo debolmente lucido, addome lucido. Corpo nero-bruno, elitre bruno-rossicce, antenne nere, zampe giallo-rossicce. Secondo antennomero più corto del primo, terzo più corto del secondo, antennomeri quarto a sesto più lunghi che larghi, settimo a nono lunghi quanto larghi, decimo trasverso. Occhi lunghi quanto le tempie. Reticolazione dell'avancorpo forte, quella dell'addome molto trasversa e molto superficiale. Granulosità di capo e pronoto fine ed evidente, assente sulla fascia longitudinale mediana del capo. Granulosità delle elitre superficiale. Una carena mediana sul primo urotergo libero basale del $\sigma^{*}$. Quinto urotergo libero del $\sigma^{*}$ con un rilievo mediano posteriore coperto di rughe longitudinali e di reticolazione forte. Edeago figg. 69-70.

\section{Comparazioni:}

Per la forma dell'edeago la nuova specie è nettamente distinta da N. smetanai PACE, 1998 della Cina. La parte preapicale dell'edeago della nuova specie, in visione laterale, è convessa mentre in smetanai è concava. L'armatura genitale interna dell'edeago della nuova specie è lunga e forte, mentre è breve ed evanescente in smetanai.

\section{Etimologia:}

La nuova specie prende nome dal Monte Erlang.

\section{Nepalota yunnanensis n. sp.}

(Figg. 16 e 71-73)

\section{Materiale tipico:}

Holotypus $0^{\star}$, China: N-Yunnan, Zhongdian Co., pass $28 \mathrm{~km}$ ESE Zhongdian, 27\%43.9' N 995․ㄹ' E, 3700-3750 m, 22.VIII.2003, leg. A. Smetana (SDEI).

Paratypus: 1 , stessa provenienza (MNHU).

\section{Descrizione:}

Lunghezza 4,1 mm. Corpo lucido e nero, elitre brune, antenne nere con i due antennomeri basali bruno-rossicci, zampe giallo-rossicce. Secondo antennomero più corto del primo, terzo più lungo del secondo, antennomeri quarto a ottavo più lunghi che larghi, nono lungo quanto largo, decimo trasverso. Occhi lunghi quanto le tempie. Reticolazione di capo e pronoto forte, quella delle elitre evidente. Punteggiatura del capo poco visibile. Granulosità del pronoto superficiale. Una lieve rugosità delle elitre nasconde una punteggiatura indistinta. Primo urotergo libero basale del $\sigma^{*}$ con superficiale carena mediana. Edeago figg. 71-72, spermateca fig. 73.

\section{Comparazioni:}

Per la forma dell'edeago la nuova specie è affine a $N$. franzi PACE, 1987 del Nepal. Se ne distingue per un'accentuata sinuosità preapicale, in visione laterale (debole sinuosità in franzi), e per i lobi apicali, in visione ventrale, dello stesso edeago assai stretti (molto larghi in franzi).

\section{Etimologia:}

La nuova specie prende nome dallo Yunnan. 


\section{Nepalota qinlingmontis n. sp.}

(Figg. 17 e 74-76)

\section{Materiale tipico:}

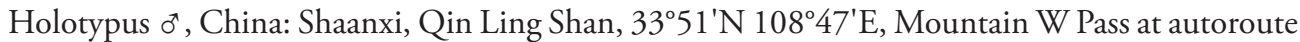
Km 70, 47 km S Xian, 2300-2500 m, sifted, 23-30.IX.1995, leg. A. Pütz (SMTD).

Paratypi: $2 o^{\star} o^{\star}$, China: Shaanxi, Qin Ling Shan, 3351'N 108 $47^{\prime} \mathrm{E}$, Mountain W Pass at autoroute $\mathrm{Km}$ 70, $47 \mathrm{~km}$ S Xian, 2300-2500 m, sifted, 26-27.IX.1995, leg. A. Pütz (SMTD); 1 q, China: Sichuan, Gongga Shan, Hailougou glacier park, above camp III, 3000 m, 30.V.1997, sifted, leg. A. Pütz (SMTD); 1 ㅇ, China: Shaanxi, Qin Ling Shan, Hua Shan, 1950-2000 m, $34^{\circ} 25^{\prime} \mathrm{N} 110^{\circ} 06^{\prime} \mathrm{E}, 19 . \mathrm{VIII} .1995$, sifted, leg. A. Pütz (SMTD).

\section{Descrizione:}

Lunghezza 3,9 mm. Corpo lucido e rossiccio, capo bruno, elitre giallo-brune, antenne brune con i due antennomeri basali giallo-rossicci, zampe giallo-rossicce. Secondo antennomero più corto del primo, terzo più lungo del secondo, antennomeri quarto a sesto più lunghi che larghi, settimo lungo quanto largo, ottavo a decimo trasversi. Occhi più corti delle tempie. Reticolazione di capo e pronoto forte, quella delle elitre evidente, quella dell'addome molto trasversa e assai superficiale, a tratti cancellata. Sesto urotergo libero del $o^{*}$ inciso al margine posteriore che è in tal modo composto di due lobi semicircolari. Edeago. figg. 74-75, spermateca fig. 76.

\section{Comparazioni:}

Per la forma dell'edeago la nuova specie è affine a N. franzi PACE, 1987 del Nepal. Se ne distingue per l'alta sinuosità preapicale ventrale dell'edeago, in visione laterale.

\section{Etimologia:}

Il nome della nuova specie significa «Del Monte Qinling».

\section{Nepalota ruficollis n. sp.}

(Figg. 18 e 77-79)

\section{Materiale tipico:}

Holotypus ơ, China, Guangxi, Nongxin, 1020 m, 12.VIII.1998, leg. R. Fellowes (IRSNB).

Paratypi: 1 ơ $^{\star}$, stessa provenienza; 1 ㅇ, China, Guangxi, Maoershan, 23.VIII.1998, leg. R. Fellowes (MNHU).

\section{Descrizione:}

Lunghezza $3 \mathrm{~mm}$. Corpo lucido e rossiccio, capo nero, pronoto e pigidio giallo-rossicci, addome bruno-rossiccio, antenne giallo-rossicce, zampe gialle. Secondo antennomero più corto del primo, terzo più lungo del secondo, antennomeri quarto a ottavo più lunghi che larghi, nono a decimo lunghi quanto larghi. Occhi lunghi quanto le tempie. Reticolazione di capo e pronoto molto superficiale, quella di elitre e addome assente. Punteggiatura del capo superficiale e diradata sul disco, quella delle elitre fitta e poco profonda. Granulosità del pronoto fine e superficiale. Pronoto con debolissimo solco mediano e superficiale e fossetta mediana posteriore. Edeago figg. 77-78, spermateca. fig. 79 . 


\section{Comparazioni:}

La nuova specie è la prima nota del genere ad avere il pronoto giallo-rossiccio. Per la forma della spermateca è simile a $N$. globulifera PACE, 1998 dello Yunnan, ma l'introflessione apicale del bulbo distale della spermateca è largo e profondo nella nuova specie, mentre è appena percettibile in globulifera. La parte prossimale della spermateca della nuova specie è corta, mentre è lunga in globulifera.

\section{Etimologia:}

Il nome della nuova specie significa «Pronoto rossiccio».

\section{Nepalota rougemonti $\mathrm{n} . \mathrm{sp}$.}

(Figg. 19 e 80-81)

\section{Materiale tipico:}

Holotypus ơ, China, Guangxi, Diding, 1160 m, 9.VII.1999, leg. R. Fellowes (IRSNB).

\section{Descrizione:}

Lunghezza 2,6 mm. Corpo lucido e giallo-rossiccio, capo nero, elitre brune con base brunorossiccia, quarto urotergo libero bruno, antenne rossicce con primo antennomero basale e metà apicale dell'undicesimo gialli, zampe gialle. Secondo antennomero più corto del primo, terzo più corto del secondo, antennomeri quarto a settimo più lunghi che larghi, ottavo lungo quanto largo, nono e decimo debolmente trasversi. Occhi più corti delle tempie. Reticolazione di capo e pronoto superficiale, quella delle elitre poco visibile. Punteggiatura di capo ed elitre superficiale. Granulosità del pronoto evanescente. Edeago figg. 80-81.

\section{Comparazioni:}

Per la forma dell'edeago, figg. 77-78 e 80-81, la nuova specie si distingue da $N$. ruficollis n. sp. sopra descritta. Per il colore del pronoto si distingue dalle specie note. In visione ventrale, l'edeago della nuova specie ha apice diviso in due stretti e corti lobi, mai osservati in altre specie del genere.

\section{Etimologia:}

La nuova specie è dedicata al noto studioso di Staphylinidae Guillaume de Rougemont, che me l'ha affidata in studio.

\section{Nepalota robusta $\mathrm{n}$. sp.}

(Figg. 20 e 82-83)

\section{Materiale tipico:}

Holotypus $0^{\star}$, China: W-Hubei, Daba Shan mtn. range NE Muyuping, creek valley, $4 \mathrm{~km} \mathrm{~N}$ Muyuping, 1700 m, 21.VII.2001, leg. A. Smetana (SDEI).

\section{Descrizione:}

Lunghezza 4,2 $\mathrm{mm}$. Corpo lucido e giallo-bruno, capo e uroterghi liberi quarto e quinto neri, antenne brune con i due antennomeri basali e base del terzo rossicci, zampe giallo-rossicce. 
Secondo antennomero più corto del primo, terzo più lungo del secondo, antennomeri quarto a decimo più lunghi che larghi. Occhi più corti delle tempie. Reticolazione dell'avancorpo forte, quella dell'addome assente. Granulosità di capo e pronoto fine e poco distinta, quella delle elitre evidente. Primo urotergo libero basale del $\sigma^{\star}$ con carena mediana posteriore saliente, secondo con rilievo mediano superficiale distante sia dal margine posteriore che da quello anteriore. Quinto urotergo libero del $\sigma^{*}$ con granuli fitti e salienti sulla fascia longitudinale mediana, sul resto dell'urotergo granuli radi. Edeago figg. 82-83.

\section{Comparazioni:}

Per la forma dell'edeago la nuova specie è comparabile con N. smetanai PACE, 1998 del Sichuan. Se ne distingue per il profilo ventrale dell'edeago sinuato (arcuato in smetanai), per i lobi apicali dell'edeago, in visione ventrale, larghi e rettilinei (stretti e arcuati in smetanai) e per la differente forma dell'armatura genitale interna dell'edeago. La reticolazione delle elitre è forte nella nuova specie, molto superficiale in smetanai.

\section{Etimologia:}

La nuova specie è detta «Robusta», a motivo del forte edeago, se comparato con quello gracile di altre specie del genere.

\section{Nepalota gracilis n. sp.}

(Figg. 21 e 84-85)

\section{Materiale tipico:}

Holotypus $0^{\star}$, China: W-Hubei, Daba Shan mtn. range NE Muyuping, creek valley, $4 \mathrm{~km} \mathrm{~N}$ Muyuping, 1700 m, 21.VII.2001, leg. A. Smetana (SDEI).

\section{Descrizione:}

Lunghezza 3,6 mm. Corpo nero, elitre nero-brune, uroterghi liberi quarto e quinto neri con riflessi iridescenti, antenne nero-brune con i due antennomeri basali e base del terzo rossicci, zampe giallo-rossicce. Secondo antennomero più corto del primo, terzo più lungo del secondo, quarto più lungo che largo, quinto e sesto lunghi quanto larghi, antennomeri settimo a decimo trasversi. Occhi lunghi quanto le tempie. Reticolazione dell'avancorpo forte, quella dell'addome assente. Granulosità del capo fine e assente sulla fascia longitudinale mediana, quella del pronoto saliente e quella delle elitre superficiale. Primo urotergo libero del $\sigma^{\star}$ con carena mediana posteriore saliente, quinto con un'area mediana coperta di fitti granuli, con il resto della superficie con granuli fini. Edeago figg. 84-85.

\section{Comparazioni:}

L'edeago della nuova specie è molto stretto all'apice, in visione ventrale, come in $N$. chinensis PACE, 1998 dello Zhetiang, Yunnan e Shanxi. La nuova specie si distingue da questa specie per l'edeago minore, lungo $0,6 \mathrm{~mm}$, invece di $0,7 \mathrm{~mm}$ come in chinensis, per l'apice dell'edeago, in visione laterale, stretto e rettilineo (largo e sinuato in chinensis) e per la forma differente dell'armatura genitale interna dell'edeago, difficilmente descrivibile. Gli antennomeri settimo a decimo sono trasversi nella nuova specie, più lunghi che larghi in chinensis.

\section{Etimologia:}

La nuova specie prende nome dalla forma del suo edeago. 


\section{Nepalota dabamontis n. sp.}

(Figg. 22 e 86-87)

\section{Materiale tipico:}

Holotypus $\sigma^{\star}$, China: W-Hubei, Daba Shan mtn. range NE Muyuping, creek valley, $4 \mathrm{~km} \mathrm{~N}$ Muyuping, 1700 m, 21.VII.2001, leg. A. Smetana (SDEI).

\section{Descrizione:}

Lunghezza 3,1 mm. Corpo lucido e bruno, margine posteriore dei tre uroterghi liberi basali e pigidio rossicci, antenne brune con i tre antennomeri basali giallo-rossicci, zampe giallo-rossicce. Secondo antennomero più corto del primo, terzo più corto del secondo, quarto lungo quanto largo, quinto a decimo trasversi. Occhi più corti delle tempie. Reticolazione di capo e pronoto molto superficiale, quella delle elitre evidente e quella dell'addome assente. Punteggiatura del capo molto evanescente. Granulosità di pronoto ed elitre superficiale. Edeago figg. 86-87.

\section{Comparazioni:}

L'armatura genitale interna dell'edeago della nuova specie è composta da tre lunghi pezzi di cui due falciformi. Simili pezzi non sono presenti nell'armatura genitale interna dell'edeago delle specie note.

\section{Etimologia:}

Il nome della nuova specie significa «Del Monte Daba».

\section{Nepalota caluoensis n. sp.}

(Figg. 24 e 91)

\section{Materiale tipico:}

Holotypus + , China: W-Sichuan, Yảan Prefecture, Shimian Co., Xiaoxiang Ling, side valley ab. Nanya Cun, nr. Caluo, 11 km S Shimian, 1250 m, 7.VII.1999, leg. A. Pütz (SMTD).

Paratypus: 1 \% , stessa provenienza (SMTD).

\section{Descrizione:}

Lunghezza 3,63 mm. Corpo lucido e bruno-rossiccio, capo e uroterghi liberi quarto e base del quinto bruni, antenne brune con i due antennomeri basali e base del terzo giallo-rossicci, zampe giallo-rossicce. Secondo antennomero lungo quanto il primo, terzo più lungo del secondo, antennomeri quarto e quinto più lunghi che larghi, sesto e settimo lunghi quanto larghi, ottavo a decimo trasversi. Occhi più corti delle tempie. Reticolazione di capo e pronoto molto superficiale, quella delle elitre trasversa e forte, quella dell'addome assente. Punteggiatura del capo molto superficiale, quella di pronoto e addome fine. Granulosità delle elitre fine e fitta. Spermateca fig. 91.

\section{Comparazioni:}

La grande lunghezza della spermateca permette di comparare la nuova specie a $N$. pernitida (PACE, 1984) di Burma e Nepal che presenta simile lunghezza della spermateca. La nuova specie se ne distingue per la debole introflessione apicale del bulbo distale della spermateca (profonda in pernitida) e per il bulbo prossimale della spermateca molto sviluppato (poco sviluppato in pernitida).

\section{Etimologia:}

La nuova specie prende nome dal toponimo Caluo. 
Nepalota daxuensis n. sp.

(Figg. 25 e 92)

\section{Materiale tipico:}

Holotypus + , China: W-Sichuan, Yảan Prefecture, Shimian Co., Daxue Shan, road btw. Anshunchang-Wanba, 12 km W Shimian, 1300 m, 9.VII.1999, leg. A. Pütz (SMTD).

\section{Descrizione:}

Lunghezza 3,63 mm. Corpo lucido e bruno-rossiccio, capo e uroterghi liberi quarto e quinto bruni, antenne brune con i due antennomeri basali e base del terzo giallo-rossicci, zampe giallorossicce. Secondo antennomero lungo quanto il primo, terzo più lungo del secondo, quarto più lungo che largo, antennomeri quinto e sesto lunghi quanto larghi, settimo a decimo debolmente trasversi. Occhi più corti delle tempie. Reticolazione di capo, pronoto e addome assente, quella delle elitre trasversa e forte. Punteggiatura del capo molto superficiale, quella di pronoto e addome fine ed evidente. Granulosità delle elitre fine e saliente. Spermateca fig. 92.

\section{Comparazioni:}

La nuova specie per la forma della spermateca è distinta dalle specie note, a motivo della parte prossimale non sinuata come in tutte le Nepalota note, ma semplicemente ricurva. Il bulbo distale della spermateca della nuova specie è ellittico, mentre in altre specie è cilindrico o subsferico.

\section{Etimologia:}

Il nome della nuova specie deriva dal Monte Daxue.

\section{Nehemitropia LoHSE, 1971}

\section{Nebemitropia lividipennis (MANNERHEIM, 1830)}

Oxypoda lividipennis Mannerheim, 1830: 79

Staphylinus sordidus MARSHAM, 1802: 514

Nehemitropia sordida; LOHSE, 1974: 103

Nehemitropia lividipennis; SMETANa, 2004: 412

2 es., China: Shaanxi, Qin Ling Shan, 3400'N 108 49'E, autoroute km 33, 30 Km SSW Xian, riverbank, 600 m, 31.VIII.1995, leg. A. Pütz; 2 es., China: Shaanxi, Wei He, bank at bridge on autoroute to Xian Airport, $15 \mathrm{~km}$ N Xian, $400 \mathrm{~m}, 34^{\circ} 24^{\prime} \mathrm{N} 108^{\circ} 55^{\prime} \mathrm{E}, 22$. VIII.1995, leg. A. Pütz; 1 es., China: Shaanxi, Qin Ling Shan, Hua Shan, $1950-2000 \mathrm{~m}, 34^{\circ} 25^{\prime} \mathrm{N} 110^{\circ} 06^{\prime} \mathrm{E}$, 19.VIII.1995, sifted, leg. A. Pütz. China: Shaanxi, Qin Ling Shan, Hua Shan, 1950-2000 m, $34^{\circ} 25^{\prime}$ N $110^{\circ} 06$ 'E, 19.VIII.1995, sifted, leg. A. Pütz; 6 es., China: N-Yunnan, Dali Bai Nat. Aut. Pref., Diancang Shan, 3 km W Dali, 2541.9'N 10008.4'E, 3.IX.2003, leg. A. Smetana (SMTD) (MNHU) (IRSNB).

\section{Distribuzione:}

Specie cosmopolita, già nota per la Cina. 
Pelioptera KraAtz, 1857

Pelioptera (Pelioptera) testaceipennis (Motschulsкy, 1858)

Homalota testaceipennis Motschulsky, 1858: 251

Homalota pelioptera KraATZ, 1859: 30; SaWAda 1980: 51

Pelioptera testaceipennis; Sawada 1980: 51; Pace 1984: 428; Smetana, 2004: 415

$1 \mathrm{o}^{\star}$, China, Sichuan, Qingcheng Shan, 65 km NW Chengdu, 3053'N 10333'E, 800-1000 m, 18.V/3-4.VI.1999 m, 8 km W Taiping.VI.1997, leg. A. Pütz (SMTD).

\section{Distribuzione:}

Specie presente nello Sri Lanka, Nepal, India, Birmania, Sabah e Giappone. Già nota per la Cina.

\section{Pelioptera (Pelioptera) opaca KraATZ, 1857}

Pelioptera opaca Kratz, 1857: 56; Cameron 1939: 418

$10^{\star}$, China: W-Sichuan, Ya’an Prefecture, Shimian Co., Xiaoxiang Ling, 7 km S Liziping, 35 km S Shimian 1600 m, $18^{\circ} 56^{\prime} \mathrm{N} 102^{\circ} 18^{\prime} \mathrm{E}, 7 . V I I .1999$, leg. A. Pütz; 1 o $^{\star}$ e 1 ㅇ, China: Shaanxi, Qin Ling Shan, Li Shan nr. Lintong, $34^{\circ} 20^{\prime} \mathrm{N} 109^{\circ} 16^{\prime} \mathrm{E}, 31 \mathrm{~km}$ E Xian, dry meadows \& forest, sifted, 1000-1200 m, 23-25.VIII.1995, leg. A. Pütz.; 5 es., China: Shaanxi, Qin Ling Shan, Li Shan nr.

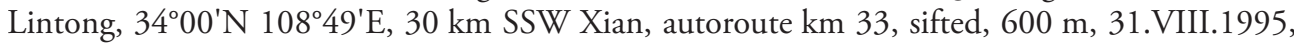
leg. A. Pütz; $1 \sigma^{\star}$, China: W-Hubei, Daba Shan pass E Mt. Shennongjie, 12 km NW Muyuping, $31^{\circ} 30^{\prime} \mathrm{N} 110^{\circ} 21^{\prime} \mathrm{E}, 1950 \mathrm{~m}, 16 . \mathrm{VII} .2001$, leg. A. Smetana; 1 o $^{\circ}$ e 1 우, China, W-Guangxi, Diding, 1110 m, 9.VII.1999, leg. R. Fellowes; 1 , China: Shaanxi, Daba Shan, creek vall SE pass $20 \mathrm{~km}$ NW Zhenping, 1700-1800 m, 31 4 44'N 109³5'E, 9.VII.2001, leg. A. Smetana; 1 ㅇ, China: Shaanxi, Daba Shan, creek vall SE pass $20 \mathrm{~km}$ NW Zhenping, $1700-1800 \mathrm{~m}, 31^{\circ} 44^{\prime} \mathrm{N}$ $109^{\circ} 35^{\prime}$ E, 12.VII.2001, leg. A. Smetana; 1 ㅇ, China: Shaanxi, Daba Shan, creek vall SE pass $20 \mathrm{~km}$ NW Zhenping, $1680 \mathrm{~m}, 31^{\circ} 59^{\prime} \mathrm{N} 109^{\circ} 22^{\prime} \mathrm{E}, 11 . \mathrm{VII} .2001$, leg. A. Smetana (SMTD) (MNHU) (IRSNB).

\section{Distribuzione:}

Specie diffusa dallo Sri Lanka all'India e a Singapore. Già nota per la Cina. Importata in Europa $\mathrm{e}$ isole atlantiche.

\section{Pelioptera (Pelioptera) nepaliella PACE, 1991}

Pelioptera (Pelioptera) nepaliella PACE, 1991: 841

1 o , China: N-Yunnan, Dali Bai Nat. Aut. Pref., Diancang Shan, 3 km W Dali, $25^{\circ} 41.1^{\prime} \mathrm{N}$ $100^{\circ} 06.6^{\prime} \mathrm{E}, 2750 \mathrm{~m}, 1 . \mathrm{IX} .2003$, leg. A. Smetana (MNHU).

\section{Distribuzione:}

Specie finora nota solo del Nepal. 
Pelioptera (Geostibida) eremita PACE, 1998

Pelioptera eremita PACE, 1998: 943

1 ㅇ , China: Hainan, Hainan, Wuzhishan, 1280 m, 9.VI.1999, 13.VI.1999, leg. J.R. Fellowes (IRSNB).

\section{Distribuzione:}

Specie finora nota solo di Hong Kong.

\section{Pelioptera (Pelioptera) uncinata PACE, 1987}

Pelioptera uncinata PACE, 1987: 408

1 ․ China: Shaanxi, Qin Ling Shan, pass rd. Zhouzhi-Foping, 105 km SW Xi'an, N-slope, 1990 m, 3344'N 10758'E, 2.VII.2001, leg. A. Smetana (MNHU).

\section{Distribuzione:}

Specie finora nota solo del Nepal.

\section{Pelioptera (Pelioptera) yunnanensis PACE, 1993}

Pelioptera yunnanensis PACE, 1993: 110

$10^{\star}$ e 2 우 옹 China: Shaanxi, Daba Shan, creek vall SE pass $20 \mathrm{~km}$ NW Zhenping, 1700-1800 m, $31^{\circ} 44^{\prime} \mathrm{N} 109^{\circ} 35^{\prime} \mathrm{E}, 9 . \mathrm{VII} .2001$, leg. A. Smetana (MNHU).

\section{Distribuzione:}

Specie finora nota solo dello Yunnan.

\section{Pelioptera (Geostibida) dalicola n. sp.}

(Figg. 26 e 93-95)

\section{Materiale tipico:}

Holotypus $0^{\star}$, China: N-Yunnan, Dali Bai Nat. Aut. Pref., Diancang Shan, $3 \mathrm{~km} \mathrm{~W} \mathrm{Dali,}$ 2541.1'N 10006.6'E, 2600-2650 m, 30.VIII.2003, leg. A. Smetana (SDEI).

Paratypus: 1 ㅇ, stessa provenienza.

\section{Descrizione:}

Lunghezza 3,1 mm. Corpo lucido e bruno, capo e uroterghi liberi quarto e quinto neri, antenne brune con antennomero basale nero-bruno e apice distale dell'undicesimo giallo sporco, zampe gialle. Secondo antennomero più corto del primo, terzo più lungo del secondo, quarto e quinto più lunghi che larghi, sesto lungo quanto largo, settimo a decimo debolmente trasversi. Occhi più lunghi delle tempie. Reticolazione di capo e pronoto superficiale, quella delle elitre evidente, quella dell'addome molto trasversa e ben visibile. Granulosità del capo evidente solo all'indietro, sul resto fine, quella delle elitre superficiale. Punteggiatura del pronoto quasi indistinta. Edeago figg. 94-95, spermateca fig. 93. 


\section{Comparazioni:}

La spermateca della nuova specie è simile a quella di P. satyrus (PACE, 1998) comb. n. (olim Geostibasoma) di Hong Kong, ma il bulbo distale della spermateca è quasi sferico nella nuova specie (cilindrico in satyrus). L'edeago della nuova specie è privo di carene ventrali, presenti al contrario in satyrus.

\section{Etimologia:}

La nuova specie ha nome che significa «Abitante di Dali».

\section{Pelioptera (Geostibida) dabamontis n. sp.}

(Figg. 27 e 96-98)

\section{Materiale tipico:}

Holotypus o ${ }^{\star}$, China: W-Hubei, Daba Shan pass E of Mt. Da Shennongjia, 12 km NW Muyuping, $31^{\circ} 30^{\prime} \mathrm{N} 110^{\circ} 21^{\prime} \mathrm{E}, 19 . \mathrm{VII} .2001,2050 \mathrm{~m}$, leg. A. Smetana (SDEI).

Paratypi: 2 우 ㅇ, stessa provenienza; 1 우, China: W-Hubei, Daba Shan creek valley, $8 \mathrm{~km}$ NW Muyuping, $31^{\circ} 29^{\prime} \mathrm{N} 110^{\circ} 22^{\prime} \mathrm{E}, 1550-1650 \mathrm{~m}, 18 . \mathrm{VII} .2001$, leg. A. Smetana; $2 \mathrm{o}^{\top} \mathrm{o}^{\top}$, China: border Shaanxi, Daba Shan pass, $20 \mathrm{~km}$ SSE Zhenping, 1700-1800 m, 31 $44^{\prime} \mathrm{N} 109^{\circ} 35^{\prime} \mathrm{E}$, 12.VII.2001, leg. A. Smetana; $10^{\star}$, China: W-Hubei, Daba Shan mtn. range NE Muyuping, creek valley, 4 km N Muyuping, 1700 m, 21.VII.2001, leg. A. Smetana (MNHU) (IRSNB).

\section{Descrizione:}

Lunghezza 2,8 mm. Corpo lucido e nero, elitre brune, antenne nere con i due antennomeri basali nero-bruni, zampe giallo-rossicce. Secondo antennomero più corto del primo, terzo più corto del secondo, quarto lungo quanto largo, quinto e sesto lunghi quanto larghi, settimo a decimo fortemente trasversi. Occhi più corti delle tempie. Reticolazione dell'avancorpo molto superficiale. Punteggiatura di capo e pronoto evidenti, sul capo assente su una stretta fascia longitudinale mediana. Granulosità delle elitre superficiale. Pronoto con una fossetta mediana basale. Edeago figg. 96-97, spermateca fig. 98.

\section{Comparazioni:}

Il profilo ventrale dell'edeago della nuova specie è simile a quello di P. kowloonensis PACE, 1999 di Hong Kong, ma la lunghezza dell'edeago è di 0,27 mm nella nuova specie, $0,18 \mathrm{~m}$ in kowloonensis. L'armatura genitale interna dell'edeago è differente nelle due specie. Gli occhi della nuova specie sono più corti delle tempie, in kowloonensis sono più lunghi delle tempie.

\section{Etimologia:}

La nuova specie ha nome che significa «Del Monte Daba».

\section{Pelioptera (Geostibida) shaanxiensis n. sp.}

(Figg. 28 e 99)

\section{Materiale tipico:}

Holotypus + , China: Shaanxi, Qin Ling Shan, pass rd. Zhouzhi-Foping, $105 \mathrm{~km} \mathrm{SW} \mathrm{Xi'an,}$ N-slope, $1990 \mathrm{~m}, 33^{\circ} 44^{\prime} \mathrm{N} 107^{\circ} 58^{\prime} \mathrm{E}$, 3.VII.2001, shady rockweall base, moist, sifted, leg. A. Smetana (SDEI). 


\section{Descrizione:}

Lunghezza 3,8 mm. Corpo lucido e nero-bruno, i tre uroterghi liberi basali giallo-bruni, antenne brune con i due antennomeri basali nero-bruni, zampe gialle. Secondo antennomero più corto del primo, terzo più corto del secondo, quarto più lungo che largo, quinto e sesto lunghi quanto larghi, settimo a decimo trasversi. Occhi più corti delle tempie. Reticolazione del capo molto superficiale, quella del pronoto forte, quella delle elitre evidente, quella dell'addome molto trasversa e molto superficiale. Punteggiatura del capo evidente. Granulosità del pronoto ben visibile come quella delle elitre. Capo con debole impressione discale. Spermateca fig. 99.

\section{Comparazioni:}

La spermateca della nuova specie è simile a quella di P. lii PACE, 1998 di Hebei Prov. Se ne distingue per il minore sviluppo della parte intermedia della stessa spermateca e per la parte prossimale flessa e non sinuata come in lii. Il quarto antennomero è più lungo che largo nella nuova specie, mentre è trasverso in lii.

\section{Etimologia:}

La nuova specie prende nome dallo Shaanxi.

\section{Pelioptera (Geostibida) asymmetrica n. sp.}

(Figg. 29 e 100)

\section{Materiale tipico:}

Holotypus ㅇ, China: N-Yunnan, Dali Bai Nat. Aut. Pref., Diancang Shan, $3 \mathrm{~km}$ W Dali, 2541.1'N 10006.8'E, 2700 m, 30.VIII-1.IX.2003, leg. A. Smetana (SDEI).

\section{Descrizione:}

Lunghezza $3 \mathrm{~mm}$. Corpo lucido e nero-bruno, uroterghi liberi terzo, quarto e quinto neri, antenne nere con primo antennomero giallo-rossiccio e secondo nero-bruno, zampe gialle. Secondo antennomero più corto del primo, terzo più corto del secondo, quarto e quinto più lunghi che larghi, sesto a decimo trasversi. Occhi più corti delle tempie. Reticolazione del capo superficiale, quella di pronoto ed elitre evidente, quella dell'addome molto trasversa ed evidente. Punteggiatura del capo superficiale. Granulosità del pronoto evanescente, quella delle elitre assente. Spermateca fig. 100 .

\section{Comparazioni:}

La spermateca della nuova specie è simile a quella di P. eremita PACE, 1998 pure della Cina. Se ne distingue per il bulbo distale asimmetrico e più sviluppato (ovale e ridotto in eremita), per l'introflessione apicale del bulbo distale larga e profonda (poco profonda in eremita) e per la parte prossimale avvolta in una spira e mezzo (senza spira in eremita). La reticolazione del pronoto della nuova specie è evidente, quella del pronoto di eremita è assente.

\section{Etimologia:}

La nuova specie prende nome di «Asimmetrica» a motivo della forma priva di simmetria del bulbo distale della spermateca. 
Tropimenelytron PACE, 1983

\section{Tropimenelytron tuberiventre (EPPELSHEIM, 1880)}

Homalota tuberiventris EpPeLSHeIM, 1880: 462

Tropimenelytron tuberiventris; PACE, 1983: 189

Tropimenelytron tuberiventre; SMETANA, 2004: 418

1 ㅇ, China: W-Sichuan, Ya'an Prefecture, Shimian Co., Xiaoxiang Ling, side valley ab. Nanya Cun, nr. Caluo, 11 km S Shimian, 1250 m, 7.VII.1999, leg. A. Pütz (SMTD).

\section{Distribuzione:}

Specie finora nota solo del Suditalia e Caucaso. Nuova per la Cina.

\section{Tropimenelytron martensianum PACE, 1987}

Tropimenelytron martensianum PACE, 1987: 407

1 오 , China: Sichuan, Gongga Shan, Hailougou glacier park, env. camp II, 2650 m, 30.V.1997, sifted, leg. A. Pütz (SMTD).

\section{Distribuzione:}

Specie finora nota solo del Nepal.

\section{Tropimenelytron viaticum PACE, 1998}

Pelioptera (Tropimenelytron) viatica PACE, 1998: 938

Tropimenelytron viaticum; SMETANA, 2004: 418

$1 \sigma^{\star}$, China: W-Hubei, Daba Shan pass E of Mt. Da Shennongjia, $12 \mathrm{~km} \mathrm{NW} \mathrm{Muyuping,}$ 19.VII.2001, 1950 m, leg. A. Smetana (MNHU).

\section{Distribuzione:}

Specie finora nota solo del Sichuan.

\section{Tropimenelytron qinlingmontis $\mathbf{n}$. sp.}

(Figg. 30 e 101-104)

\section{Materiale tipico:}

Holotypus ơ, China: Shaanxi, Qin Ling Shan, 3345'N 10756'E, autoroute Km 93 S of Zhouzhi, $108 \mathrm{Km}$ SW Xian, mountain forest, sifted, 1650 m, 1-2.IX.1995, leg. A. Pütz (SMTD).

Paratypi: 11 es., stessa provenienza (SMTD).

\section{Descrizione:}

Lunghezza 3,3 mm. Corpo lucido e giallo-bruno, capo nero, pronoto bruno, antenne brune con i due antennomeri basali giallo-bruni, zampe giallo-rossicce. Secondo antennomero più corto del primo, terzo più corto del secondo, quarto lungo quanto largo, quinto a decimo trasversi. Occhi lunghi quanto le tempie. Reticolazione del capo forte, quella del pronoto superficiale, quella delle 
elitre evidente, quella dell'addome molto trasversa e ben visibile. Granulosità del capo saliente, quella di pronoto ed elitre fitta e superficiale, quella dell'addome evidente. Edeago figg. 102-103, spermateca fig. 104, sesto urotergo libero del $\sigma^{\top}$ fig. 101.

\section{Comparazioni:}

La nuova specie presenta la parte prossimale della spermateca piegata brevemente e non descrivente due spire come in $T$. tuberiventris (EPPELSHeIm, 1879). L'edeago è lungo e non tozzo come quello di tuberiventris.

\section{Etimologia:}

Il nome della nuova specie deriva da quello del Monte Qin Ling.

\section{Berca BlackWELDER, 1952}

\section{Berca sinensis n. sp.}

(Figg. 31 e 105-108)

\section{Materiale tipico:}

Holotypus + , China: W-Sichuan, Aba Tibetian Auton. Prefecture, Weizhou Co., Qionglai Shan,

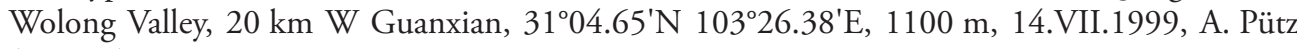
(SMTD).

Paratypus: 1 ㅇ stessa provenienza (SMTD).

\section{Descrizione:}

Lunghezza 2,33 mm. Corpo lucido e bruno, antenne nero-brune con i due antennomeri basali bruni, zampe giallo-brune. Secondo antennomero più corto del primo, terzo più corto del secondo, antennomeri quarto a decimo fortemente trasversi. Occhi più lunghi delle tempie. Reticolazione dell'avancorpo assente, quella dell'addome trasversa ed evidente. Punteggiatura del capo forte e profonda, assente sul disco e sulla fronte, quella del pronoto grossolana e profonda, quella delle elitre fitta ed evidente. Granulosità dell'addome saliente. Debole solco mediano posteriore del pronoto, confluente nella fossetta posteriore. Spermateca fig. 105, sesto urotergo libero del ${ }^{\top}$ fig. 108.

\section{Comparazioni:}

Per la forma della spermateca la nuova specie è simile a B. nepalensis PACE, 1991 del Nepal. Se ne distingue per la minore lunghezza della spermateca, $0,21 \mathrm{~mm}$, (in nepalensis $0,32 \mathrm{~mm}$ ) e per la meno profonda introflessione apicale del bulbo distale della spermateca. La reticolazione è assente sul pronoto della nuova specie, mentre sul pronoto di nepalensis la reticolazione è forte. La punteggiatura del pronoto della nuova specie è grossolana e distribuita su tutta la superficie, in nepalensis, invece, la punteggiatura del pronoto non è grossolana ed è assente sulla fascia longitudinale mediana.

\section{Etimologia:}

La nuova specie prende nome dalla Cina. 
Amphibolusa PACE, 1998

Amphibolusa smetanai PACE, 1998

Amphibolusa smetanai PACE, 1998: 458

$20^{\star} \sigma^{\star}$, China: N-Yunnan, Zhongdian Co., $10 \mathrm{~km} \mathrm{SW}$ Zhongdian, $27^{\circ} 46.5^{\prime} \mathrm{N} 98^{\circ} 36.5^{\prime} \mathrm{E}, 3800 \mathrm{~m}$, 20.VIII.2003, leg. A. Smetana (MNHU).

\section{Distribuzione:}

Specie finora nota solo del Sichuan.

\section{Amphibolusa chinensis n. sp.}

(Figg. 32 e 109-110)

\section{Materiale tipico:}

Holotypus ơ, China: W-Sichuan, Ganzi Tibet, Aut. Prefecture, Luding W Erlang Shan-Pass, $29^{\circ} 51^{\prime} \mathrm{N} 102^{\circ} 15^{\prime} \mathrm{E}, 2600$ m, Laubstreu, Pilze, 29.VI.1999, leg. M. Schülke (MNHU).

\section{Descrizione:}

Lunghezza $2 \mathrm{~mm}$. Corpo lucido e giallo rossiccio, capo e uroterghi liberi terzo, quarto e base del quinto bruni, antenne giallo-rossicce con i tre antennomeri basali gialli, zampe gialle. Secondo antennomero più corto del primo, terzo molto più corto del secondo, antennomeri quarto a decimo fortemente trasversi. Occhi più corti delle tempie. Reticolazione dell'avancorpo evidente, quella dell'addome visibile solo sulla metà posteriore di ciascun urotergo libero. Granulosità del capo superficiale, quella di pronoto ed elitre assente. Disco del capo impresso. Edeago figg. 109-110.

\section{Comparazioni:}

Per la forma della ligula e dei palpi labiali, la nuova specie va attribuita al genere Amphibolusa PACE, 1998 diffuso anche nel Laos. Il pronoto della nuova specie è poco trasverso rispetto quello di A. smetanai PACE, 1998 della Cina. La nuova specie è distinta da questa specie anche per l'edeago meno lungo, $0,2 \mathrm{~mm},(0,6 \mathrm{~mm}$ di lunghezza in smetanai) e fortemente arcuato all'apice in visione laterale (poco ricurvo in smetanai).

\section{Etimologia:}

La nuova specie prende nome dalla Cina.

\section{Gastropaga Bernhauer, 1915}

\section{Gastropaga (Rougemontia) siamensis PACE, 1984}

\section{Rougemontia siamensis PACE, 1984: 450}

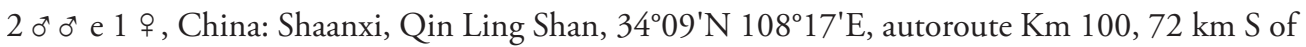
Zhouzhi, 400 m, 24.VIII.1995, leg. A. Pütz (SMTD); 1 , China: Beijing, International Airport, $40^{\circ} 08^{\prime} \mathrm{N} 116^{\circ} 35^{\prime} \mathrm{E}, 15$. VIII.1995, sifted, leg. A. Pütz (SMTD); $10^{\star}$, China: Sichuan, Gongga Shan, Hailougou glacier park, env. camp II, 2650 m, 30.V.1997, sifted, leg. A. Pütz (SMTD).

\section{Distribuzione:}

Specie finora nota solo della Thailandia. 


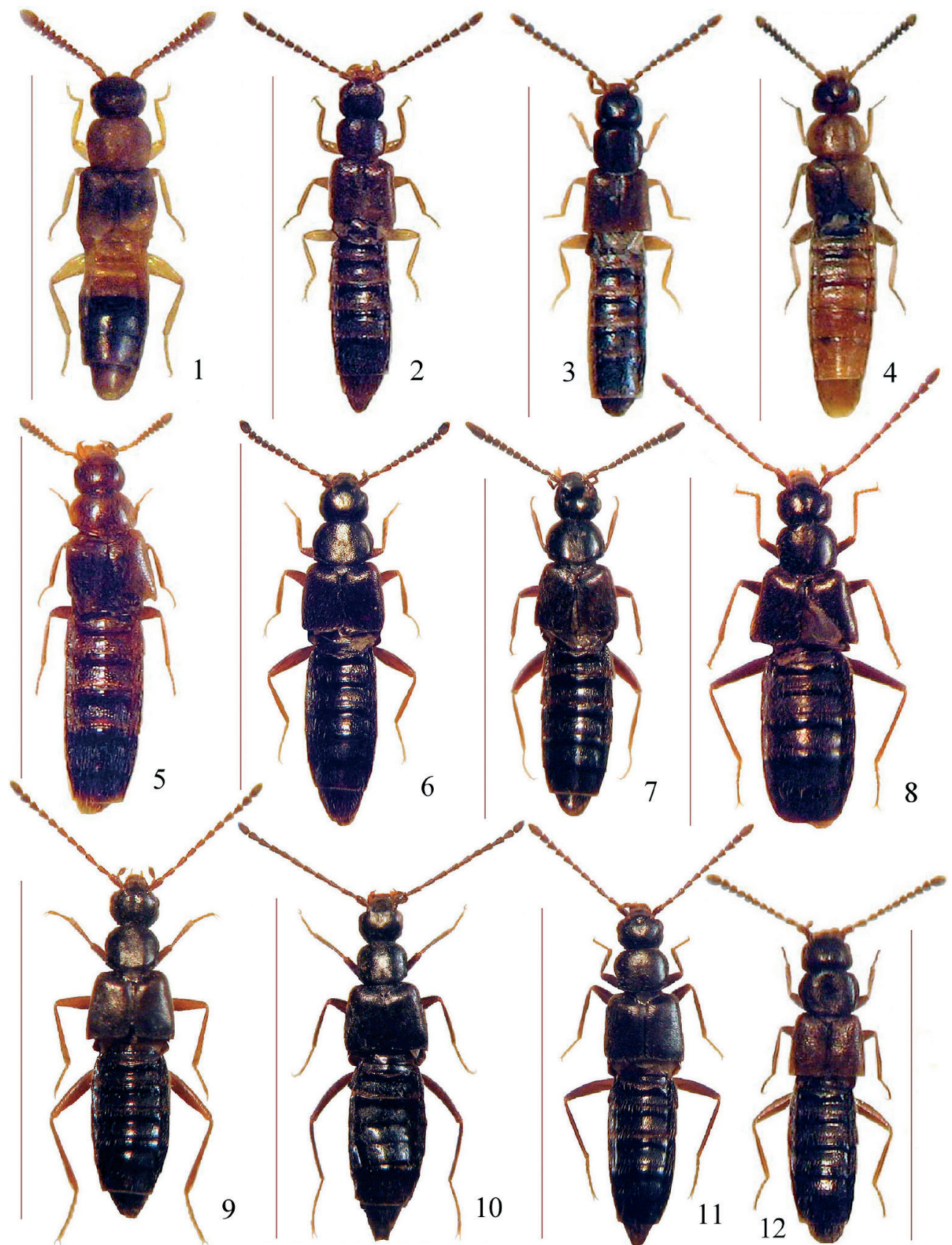

Figg. 1-12: Habitus. 1. Lasiosomina weiensis $\mathrm{n}$. sp., scala 1,6 mm; 2. Hydrosmecta sichuanensis n. sp., scala $2,3 \mathrm{~mm}$; 3. Hydrosmecta sinica $\mathrm{n}$. sp., scala 2,2 mm; 4. Amischa sichuanensis $\mathrm{n}$. sp., scala 2,1 mm; 5 . Alomaina sinica n. sp., scala 2,8 mm; 6. Paraloconota shalulimontis n. sp., scala 4,4 mm; 7. Paraloconota daxuemontis n. sp., scala 4,56 mm; 8. Paraloconota erlangensis n. sp., scala 4,24 mm; 9. Paraloconota daxuensis $\mathrm{n}$. sp., scala $3,93 \mathrm{~mm}$; 10. Paraloconota tibetana n. sp., scala $5,7 \mathrm{~mm} ; 11$. Paraloconota bilobifera n. sp., scala 3,93 mm; 12. Bellatheta tibetana $\mathrm{n}$. sp., scala $2,33 \mathrm{~mm}$. 


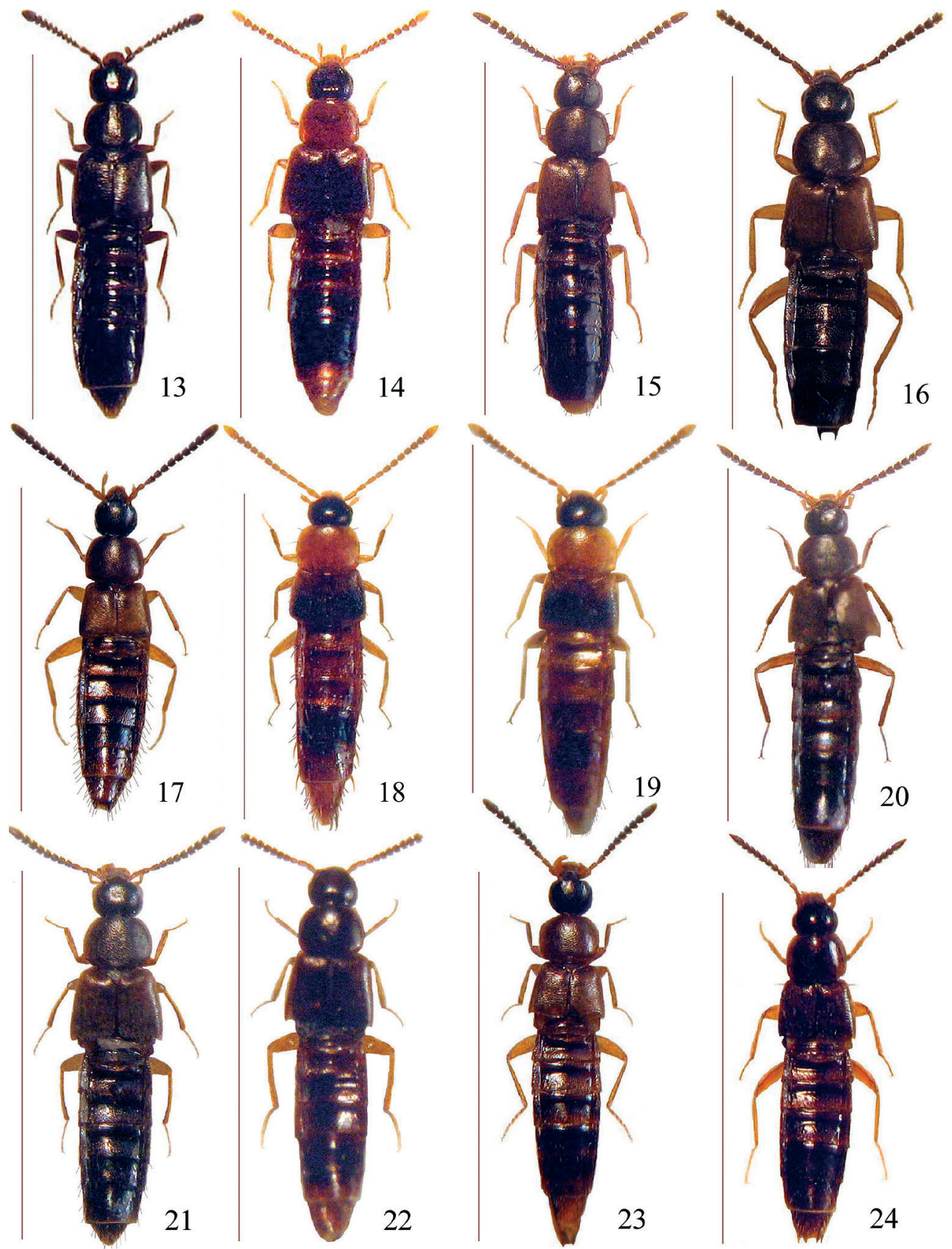

Figg. 13-24: Habitus. 13. Bellatheta huamontis n. sp., scala $2,57 \mathrm{~mm}$; 14 . Bellatheta ruficollis n. sp., scala $2,6 \mathrm{~mm}$; 15. Bellatheta yunnanensis n. sp., scala $4 \mathrm{~mm}$; 16 . Nepalota yunnanensis n. sp., scala $4,1 \mathrm{~mm}$; 17. Nepalota qinlingmontis n. sp., scala $3,9 \mathrm{~mm}$; 18. Nepalota ruficollis n. sp., scala $3 \mathrm{~mm}$; 19. Nepalota rougemonti n. sp., scala $2,6 \mathrm{~mm} ; 20$. Nepalota robusta n. sp., scala $4,2 \mathrm{~mm} ; 21$. Nepalota gracilis n. sp., scala 3,6 mm; 22. Nepalota dabamontis n. sp., scala $3,1 \mathrm{~mm}$; 23. Nepalota smetanai Pace, scala 3,48 mm; 24. Nepalota caluoensis $\mathrm{n}$. sp., scala 3,63 mm. 


\section{Ringraziamenti}

Per avermi affidato in studio il materiale oggetto del presente lavoro rivolgo i miei più cordiali ringraziamenti ai colleghi Dr. Aleš Smetana di Ottawa, Guillaume de Rougemont di London, Michael Schülke di Berlino e Olaf Jäger dello Senckenberg Museum für Tierkunde di Dresda. Per il prestito di tipi ringrazio il Dr. Peter Hammond del Museo di Storia Naturale di Londra, il Dr. Didier Drugmand de l'Institut royal des Sciences naturelles de Belgique il Dr. Lothar Zerche dello SDEI di Münchenberg, il Dr Alfred Newton del Field Museum of Natural History di Chicago e il Dr. Manfred Uhlig del Museo Zoologico dell'Università Humboldt di Berlino.

\section{Bibliografia}

Bernhauer, M. 1915: Zur Staphylinidenfauna der Philippinen: VI. Beitrag zur Kenntnis der indomalayischen Fauna. - The Philippine Journal of Science 10 (2): 117-129.

BLACKWELDER, R. E. 1952: The generic names of the beetle family Staphylinidac, with an essay on genotypy. - Bulletin of the United States National Museum 200: 483 pp.

Cameron, M. 1939: The Fauna of British India, including Ceylon and Burma. - Coleoptera, Staphylinidae 4: 410 pp., London.

Dvořák, M. 1988: Zwei neue Arten des myrmecophilen Tribus Zyrasini (Coleoptera, Staphylinidae, Aleocharinae). - Annotationes Zoologicae et Botanicae 184: 1-6.

Eppelsheim, E. 1880: [New species]. - In: Leder, H.: Beitrag zur kaukasischen Käfer-Fauna. - Verhandlungen der Kaiserlich-Königlichen Zoologisch-Botanischen Gesellschaft in Wien 29: 451-488.

KraAtz, G. 1857: Genera Aleocharinorum illustrata. - Linnaea Entomologica 11: 1-43.

Lohse, G. A. 1971: Über gattungsfremde Arten und Artenkreise innerhalb der «Grossgattung» Atheta Thomson. - Verhandlungen des Vereines für die Naturwissenschaftliche Heimatforschung Hamburg 38: $67-83$.

Lohse, G. A. 1974: Staphylinidae 11 (Hypocyphtinae und Aleocharinae). - In: Die Käfer Mitteleuropas. Band 5: 304 pp., Krefeld.

Mannerheim, C. G. 1830: Précis d'un nouvel arrangement de la Famille des Brachélytres de l'Ordre des Insectes Coléoptères. - Mémoires de l'Académie Impériale des Sciences de St. Pétersbourg 1: 415-511.

Marsham, T. 1802: Entomologia Britannica, sistens insecta Britanniae indigena, secundum methodum Linnaeanam disposita. Tomus I. Coleoptera. - Londini: Wilks \& Taylor: xxxi+ $547+$ [1] pp.

Maruyama, M. 2006: Revision of the palearctic Species of the myrmecophilous Genus Pella (Coleoptera, Staphylinidae, Aleocharinae). - National Science Museum Monograph No. 32: 207 pp., National Science Museum, Tokyo.

PACE, R. 1982a: Ocosomechusa besucheti n. gen., n. sp. dell'India ed Emmelostiba besucheti n. gen., n. sp. del Libano. - Revue suisse de Zoologie 89: 443-450.

PACE, R. 1982b: Aleocharinae del Nepal e dell'India settentrionale raccolte dal Prof. H. Franz. II. Callicerini (Coleoptera Staphylinidae) (XXXV Contributo alla conoscenza delle Aleocharinae). - Bollettino della Società entomologica italiana 114: 147-158, 49 figg.

PACE, R. 1983: Il genere Tropimenelytron Scheerpeltz (Coleoptera Staphylinidae) (XXXIX Contributo alla conoscenza delle Aleocharinae). - Nouvelle Revue d'Entomologie 13: 185-190.

PACE, R. 1984: Aleocharinae della Thailandia e della Birmania riportate da G. de Rougemont. - Bollettino del Museo civico di Storia naturale de Verona 2: 427-468.

PACE, R. 1985: Aleocharinae attere della regione australiana e neozelandese raccolte dal Prof. Franz (Coleoptera Staphylinidae) (LXIV Contributo alla conoscenza delle Aleocharinae). - Fragmenta Entomologica 18: 105-114, 37 figg. 
PACE, R. 1987a: Staphylinidae dell'Himalaya Nepalese. Aleocharinae raccolte dal Prof. Dr. J. Martens (Insecta: Coleoptera) (LXXI Contributo alla conoscenza delle Aleocharinae). - Courier ForschungsInstitut Senckenberg 93: 383-441, 210 figg.

PaCE, R. 1987b: Aleocharinae riportate dall'Himalaya dal Prof. Franz. Parte III. - Nouvelle Revue d'Entomologie 4: 117-131.

PACE, R. 1990: Aleocharinae delle Filippine. 82 Contributo alla conoscenza delle Aleocharinae. - In: Berti, N. (ed.): Miscellanées sur les Staphylins. - Mémoires du Muséum National d'Histoire naturelle (A) 147: 57-113.

PACE, R. 1991: Aleocharinae nepalesi del Museo di Ginevra. Parte V: Athetini (conclusione) e Thamiaraeini

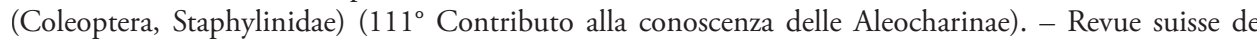
Zoologie 98: 803-863.

PACE, R. 1993: Aleocharinae della Cina (Coleoptera Staphylinidae). - Bollettino del Museo civico di Storia naturale di Verona 17: 69-126.

PACE, R. 1998: Aleocharinae della Cina: Parte II. - Revue suisse de Zoologie 105 (2): 395-463.

PACE, R. 1999: Aleocharinae di Hong Kong (Coleoptera, Staphylinidae). - Revue suisse de Zoologie 106: 663-689, 79 figg.

PaCe, R. 2004: Hygronomini e Athetini della Cina con note sinonimiche (Coleoptera, Staphylinidae). Revue suisse de Zoologie 111: 457-523, 213 figg.

Roubal, J. 1928: Zwei neue Coleopteren aus der Tschechoslovakei. - Wiener Entomologische Zeitung 45: 27-30.

Smetana, A. 2004: Aleocharinae, pp. 353-494. - In: Löвl, I. \& Smetana, A. (editors). - Catalogue of Palaearctic Coleoptera, Vol. 2. Stenstrup: Apollo Books: 942 pp.

Stephens, J. F. 1835: Illustrations of British Entomology. - Mandibulata 5: 369-448, London.

Thomson, C. G. 1858: Skandinaviens Coleoptera, synoptiskt bearbeitate. Tom III. - Lund, Berlinska Boktryckeriet, $278 \mathrm{pp}$.

Vogel, J. 2006: Bellatheta fratica (Roubal, 1928) - Ein Erstnachweis für Deutschland mit Bemerkungen zur Synonymie. - Entomologische Blätter 102: 42-47.

\section{Indirizzo dell'autore:}

Roberto Pace

Via Vittorio Veneto, 13

37032 Monteforte d'Alpone (Verona)

Italia

e-mail: pace.ent@tiscali.it

\section{Subject editor:}

Dr. L. Zerche 

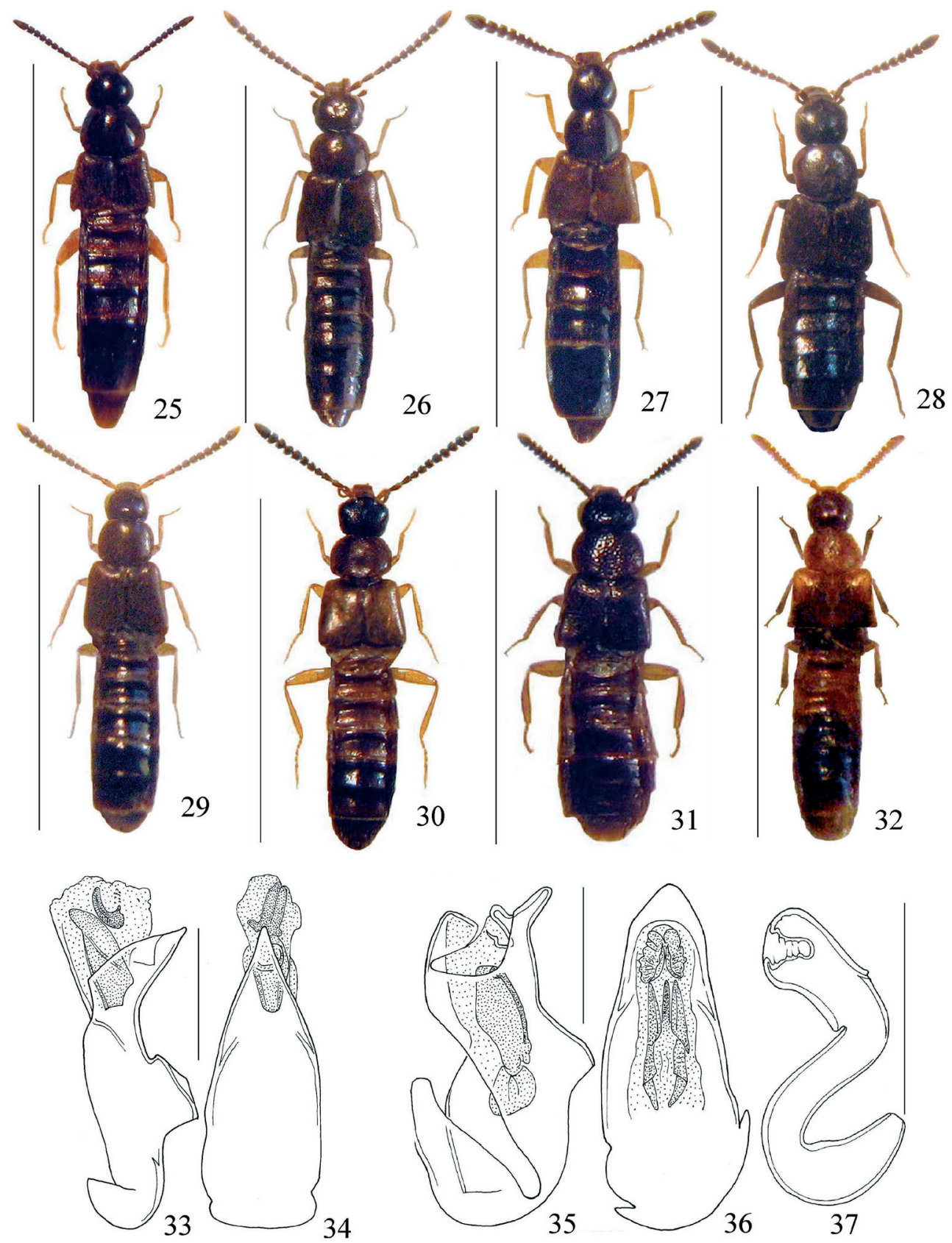

Figg. 25-37: Habitus, edeago in visione laterale e ventrale e spermateca. 25. Nepalota daxuensis n. sp., scala $3,63 \mathrm{~mm}$; 26. Pelioptera dalicola $\mathrm{n}$. sp., scala $3,1 \mathrm{~mm} ; 2$. Pelioptera dabamontis n. sp., scala $2,8 \mathrm{~mm}$; 28. Pelioptera shaanxiensis $\mathrm{n}$. sp., scala $3,8 \mathrm{~mm}$; Pelioptera asymmetrica $\mathrm{n}$. $\mathrm{sp}$., scala $3 \mathrm{~mm} ; 30$. Tropimenelytron qinlingmontis $\mathrm{n}$. sp., scala $3,3 \mathrm{~mm} ; 31$. Berca sinensis $\mathrm{n}$. sp., scala $2,33 \mathrm{~mm} ; 32$. Amphibolusa chinensis $\mathrm{n}$. sp., scala $2 \mathrm{~mm}$; 33-34. Lasiosomina weiensis n. sp.; 35-37. Hydrosmecta sichuanensis n. sp. 


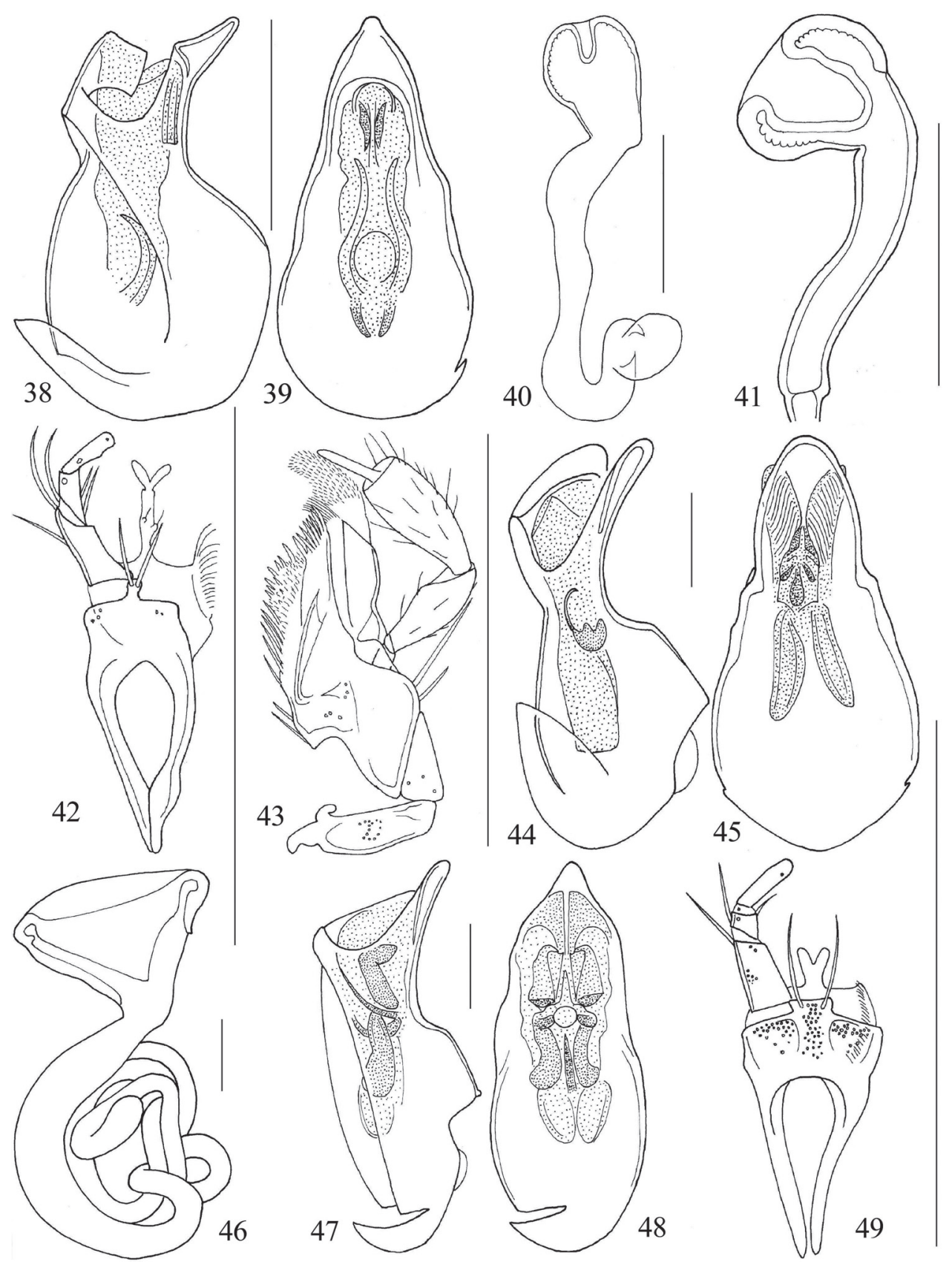

Figg. 38-49: Edeago in visione laterale e ventrale, spermateca, labio con palpo labiale, maxilla con palpo mascellare. 38-39. Hydrosmecta sinica n. sp.; 40. Amischa sichuanensis n. sp.; 41-43. Alomaina sinica n. sp.; 44-46. Paraloconota shalulimontis n. sp.; 47-48. Paraloconota daxuemontis n. sp.; 49. Paraloconota erlangensis n. sp. 


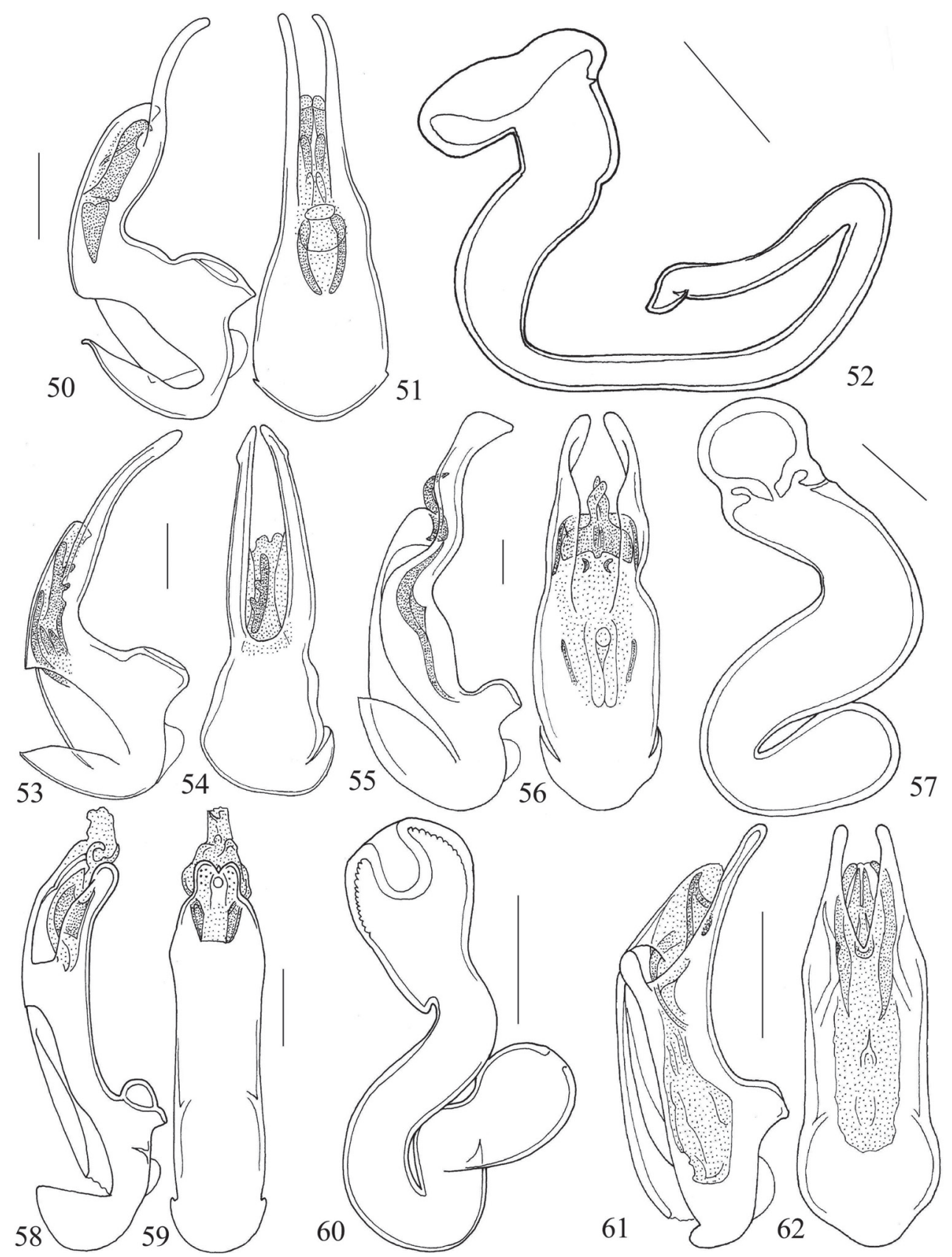

Figg. 50-62: Edeago in visione laterale e ventrale e spermateca. 50-52. Paraloconota erlangensis n. sp.; 53-54. Paraloconota daxuensis n. sp.; 55-57. Paraloconota tibetana n. sp.; 58-60. Paraloconota bilobifera n. sp.; 61-62. Bellatheta tibetana n. sp. 


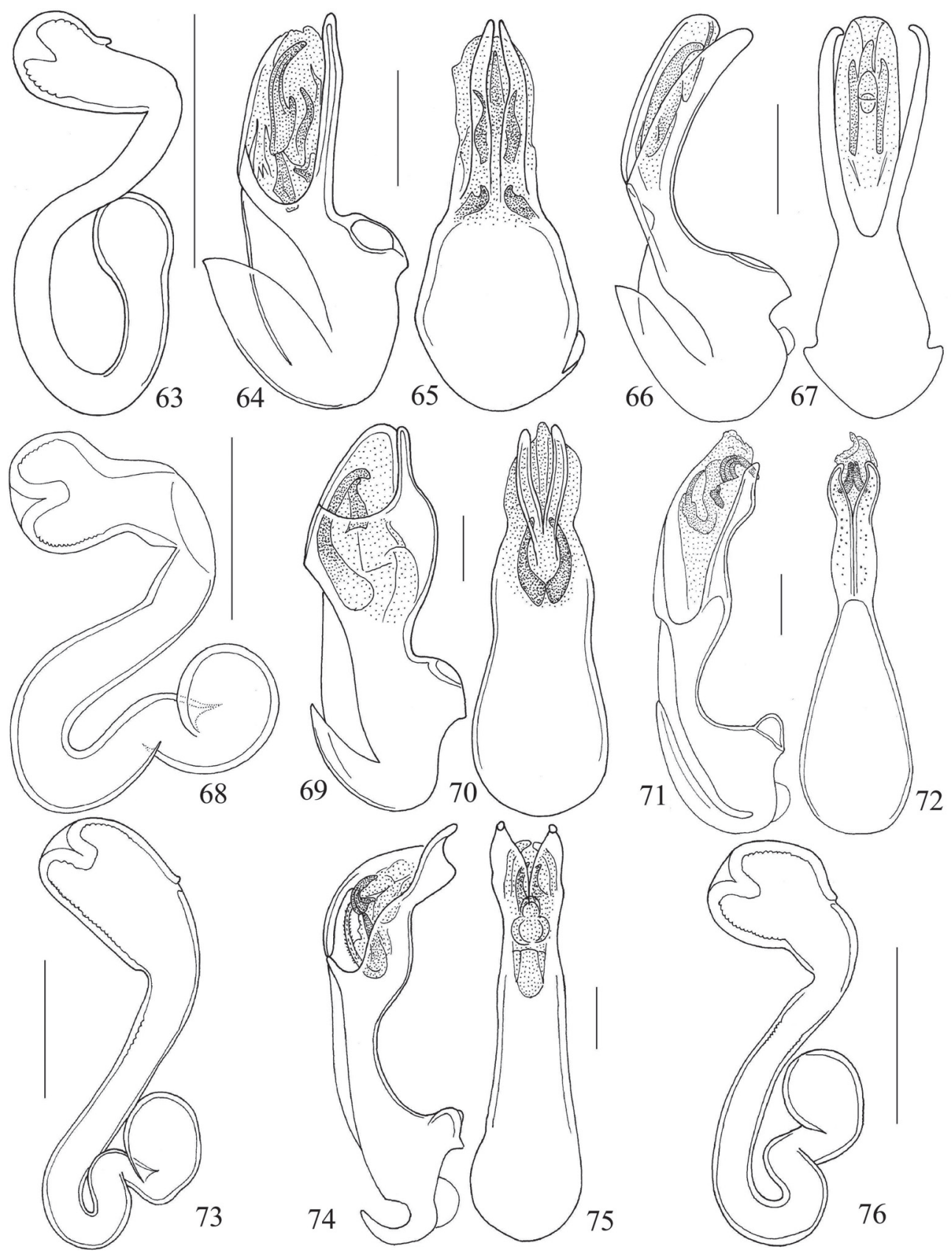

Figg. 63-76: Spermateca e edeago in visione laterale e ventrale. 63. Bellatheta tibetana n. sp.; 64-65. Bellatheta huamontis n. sp.; 66-67. Bellatheta ruficollis n. sp.; 68. Bellatheta yunnanensis n. sp.; 69-70. Nepalota erlangensis n. sp.; 71-73. Nepalota yunnanensis n. sp.; 74-76. Nepalota qinlingmontis n. sp. 

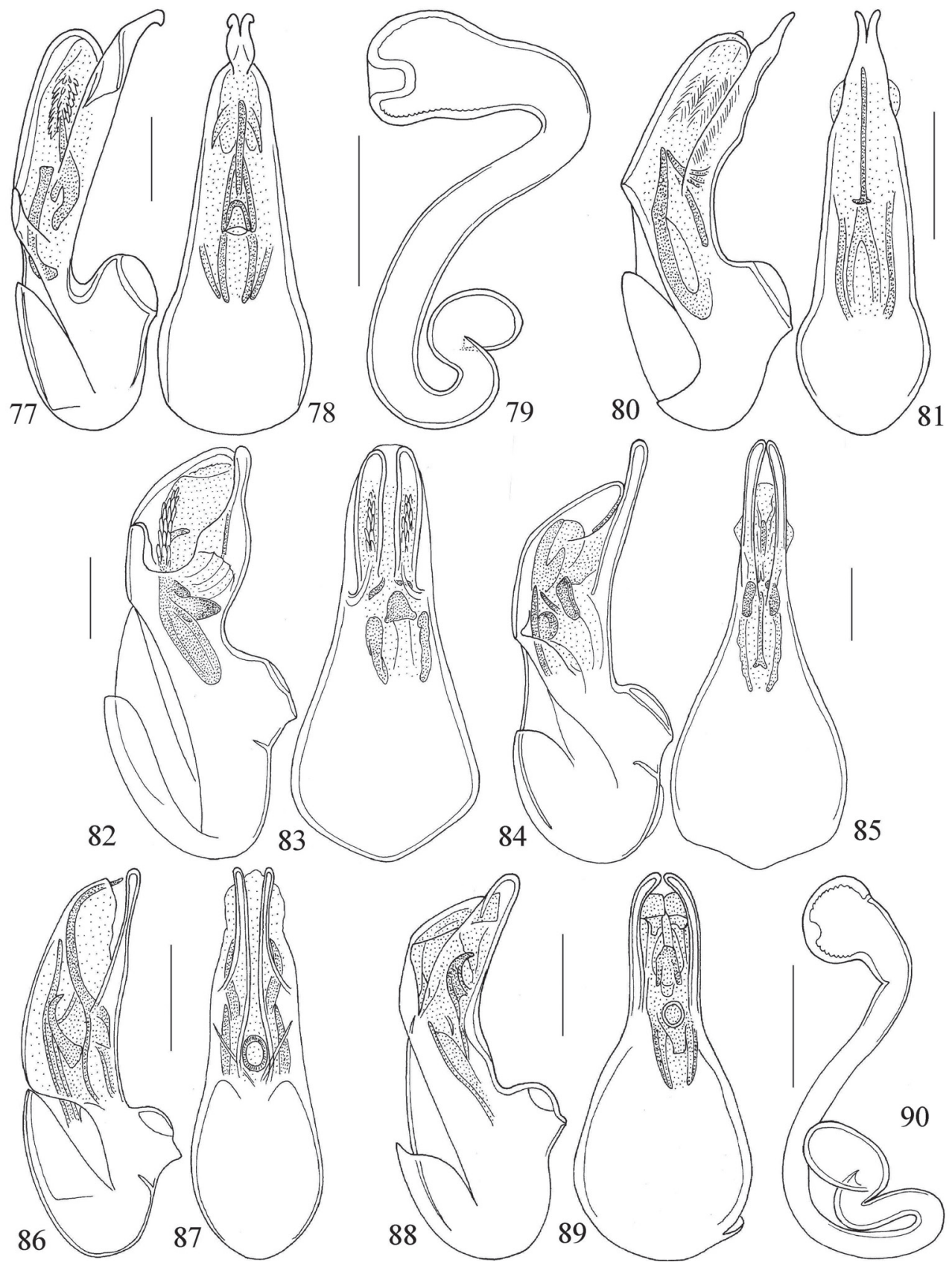

Figg. 77-90: Edeago in visione laterale e ventrale e spermateca. 77-79. Nepalota ruficollis n. sp.; 80-81. Nepalota rougemonti n. sp.; 82-83. Nepalota robusta n. sp.; 84-85. Nepalota gracilis n. sp.; 86-87. Nepalota dabamontis n. sp.; 88-90. Nepalota smetanai PACE. 


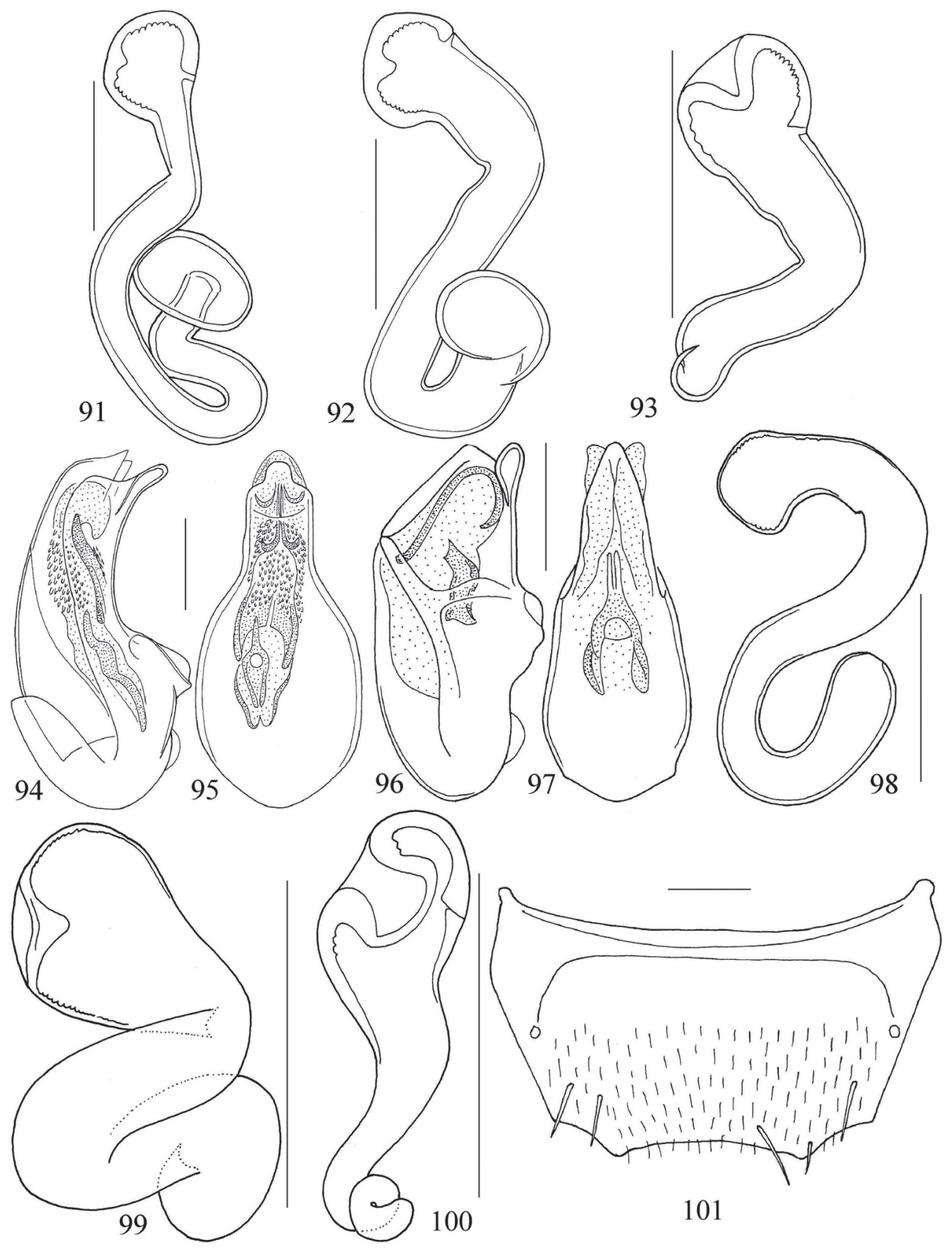

Figg. 91-101: Spermateca, edeago in visione laterale e ventrale e sesto urotergo libero della 9.91 . Nepalota caluoensis n. sp.; 92. Nepalota daxuensis n. sp.; 93-95. Pelioptera (Geostibasoma) dalicola n. sp.; 96-98. Pelioptera (Geostibasoma) dabamontis n. sp.; 99. Pelioptera (Geostibida) shaanxiensis n. sp.; 100-101. Pelioptera (Geostibida) asymmetrica n. sp. 

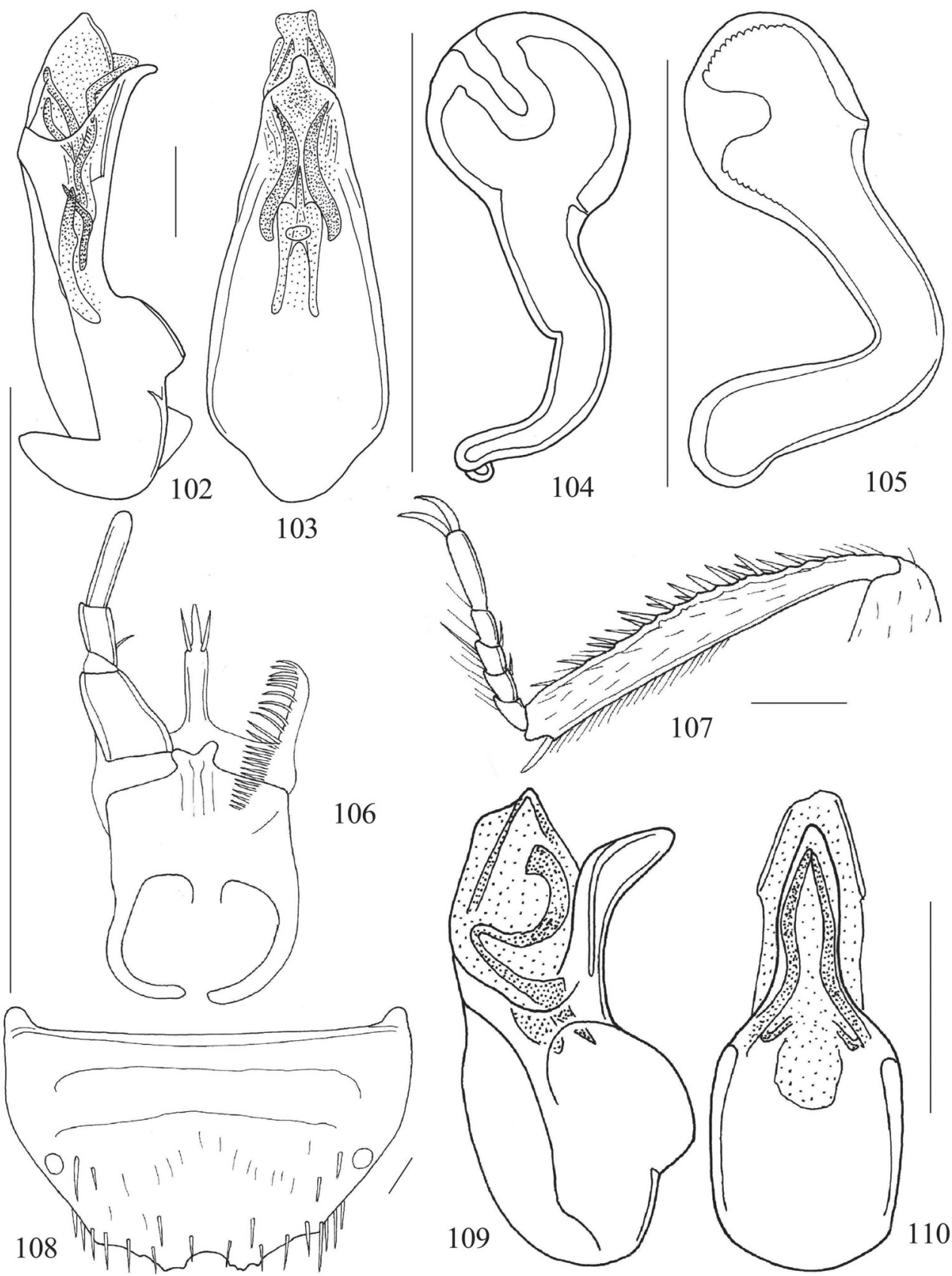

Figg. 102-110: Edeago in visione laterale e ventrale, spermateca labio con palpo labiale, mesotibia. 102-104. Tropimenelytron qinlingmontis n. sp.; 105-108. Berca sinensis n. sp.; 109-110. Amphibolusa chinensis n. sp. 